HU-EP-00/58

AEI-2000-84

hep-th/0012213

\title{
On the Vacuum Structure of Type II String Compactifications on Calabi-Yau Spaces with H-Fluxes
}

\author{
Gottfried Curio $^{a}$, Albrecht Klemm $^{a}$, Dieter Lüst ${ }^{a}$ and Stefan Theisen ${ }^{b}$ \\ ${ }^{a}$ Humboldt-Universität zu Berlin, Institut für Physik, D-10115 Berlin, Germany \\ ${ }^{b}$ Max-Planck-Institut für Gravitationsphysik, D-14476 Golm,
}

\begin{abstract}
We discuss the vacuum structure of type IIA/B Calabi-Yau string compactifications to four dimensions in the presence of $n$-form H-fluxes. These will lift the vacuum degeneracy in the Calabi-Yau moduli space, and for generic points in the moduli space, $\mathcal{N}=2$ supersymmetry will be broken. However, for certain 'aligned' choices of the H-flux vector, supersymmetric ground states are possible at the degeneration points of the CalabiYau geometry. We will investigate in detail the H-flux induced superpotential and the corresponding scalar potential at several degeneration points, such as the Calabi-Yau large volume limit, the conifold loci, the Seiberg-Witten points, the strong coupling point and the conformal points. Some emphasis is given to the question whether partial supersymmetry breaking can be realized at those points. We also relate the H-flux induced superpotential to the formalism of gauged $\mathcal{N}=2$ supergravity. Finally we point out the analogies between the Calabi-Yau vacuum structure due to H-fluxes and the attractor formalism of $\mathcal{N}=2$ black holes.
\end{abstract}

December 2000

\footnotetext{
${ }^{1}$ email: aklemm, curio,luest@physik.hu-berlin.de, theisen@aei-potsdam.mpg.de
} 


\section{Introduction}

In this paper we will discuss the vacuum structure of type II strings on Calabi-Yau threefolds with internal $n$-form H-fluxes turned on. In general, the effect of non-vanishing H-fluxes is that they lift the vacuum degeneracy in the Calabi-Yau moduli space. In fact, as already discussed in 11, 2, 3, 4, at generic points in the Calabi-Yau moduli space, nontrivial Ramond and/or Neveu-Schwarz $n$-form H-fluxes generally break $\mathcal{N}=2$ space-time supersymmetry completely, unless their contribution to the vacuum energy is balanced by other background fields, such as the dilaton field in heterotic string compactifications [5]. However $\mathcal{N}=2$ or $\mathcal{N}=1$ supersymmetric vacua can be found at certain corners in the moduli space, where the Calabi-Yau geometry is degenerate. We will consider several degeneration points of the Calabi-Yau geometry, such as the large volume limit, the Calabi-Yau conifold point, the Seiberg-Witten limit and the strong coupling singularity. However as soon as one abandons these special points, supersymmetry will be in general broken. E.g. going away from the classical large radius limit, type IIA world-sheet instanton corrections to the prepotential imply a non-degenerate period vector such that supersymmetry gets broken [1]. In addition, there might be the possibility for unbroken supersymmetry in case the contribution of the Ramond fluxes is balanced by the NSfluxes, as we will discuss at the end of the paper.

Turning on $n$-form H-fluxes on the six-dimensional Calabi-Yau space corresponds to a gauging of certain hypermultiplet isometries in the low-energy $\mathcal{N}=2$ supergravity action and leads to a non-vanishing scalar potential in four dimensions which lifts the previous vacuum degeneracy [1, 2]. Alternatively the H-fluxes can be described by a non-trivial superpotential $W$ in four dimensions, which is expressed in terms of $\mathcal{N}=1$ chiral fields [3]. Specifically, it turns out that the superpotential is simply given by the symplectic scalar product of the (dilaton dependent) H-flux vector with the $\mathcal{N}=2$ period vector $\Xi$, which is a function of the complex scalars residing in the $\mathcal{N}=2$ vector multiplets. In this way the superpotential is closely tied up to the Calabi-Yau geometry, since the period vector $\Xi$ corresponds to the various geometric cycles of the Calabi-Yau space. The question of supersymmetry breaking is then intimately related to the question whether the H-fluxes are turned on in the directions of the vanishing cycles of the Calabi-Yau spaces (aligned case) or not (misaligned case). For the aligned situations, the degeneration points in the Calabi-Yau geometry are attractor points where supersymmetry is unbroken and the potential exhibits a (local) minimum of zero energy. On the other hand, in case of H-fluxes which are misaligned with respect to a particular vanishing cycle, supersymmetry will be

\footnotetext{
${ }^{2}$ Related types of Calabi-Yau superpotentials were discussed before in [6].
} 
broken at the degeneration points in the Calabi-Yau geometry. Therefore the question which degeneration point corresponds to a supersymmetric ground state depends crucially on the chosen H-fluxes.

In this paper we will first show that the gauging of $\mathcal{N}=2$ supergravity due to H-fluxes leads to the superpotential of [3]. Subsequently we will discuss in detail the vacuum structure of type II Calabi-Yau compactifications with H-flux induced superpotential. The paper is organized as follows. In the next section we shortly review those aspects of $\mathcal{N}=2$ special geometry, which we need for our discussion, as well as the derivation of the symplectic invariant superpotential from the gauged hypermultiplet couplings. Analyzing the structure of the gravitino mass matrix which follows from the H-flux induced superpotential we will see that partial supersymmetry breaking from $\mathcal{N}=2$ to $\mathcal{N}=1$ supersymmetry $[7,8]$ is a priori possible in case the flux vector is complex which means that Ramond as well as NS fluxes have to be turned on. However treating the type IIB dilaton field as a dynamical variable $\mathcal{N}=2$ supersymmetry will be either unbroken or completely broken at the minimum of the scalar potential. Therefore at the degeneration points with aligned fluxes in the Calabi-Yau geometry, the full $\mathcal{N}=2$ supersymmetry is unbroken. Finally, at the end of sect. 2, we point out that the superpotential formalism due to internal H-fluxes is closely related to $\mathcal{N}=2$ black holes and the so called attractor formalism [9, 10]. In fact, when computing the supersymmetric points in the effective supergravity action one has to solve precisely the same equations which determine the scalar fields at the horizon of the $\mathcal{N}=2$ black holes. This means that the supersymmetric ground states with H-fluxes are the attractor points of the $\mathcal{N}=2$ black holes.

In sect. 3 we discuss in detail the vacuum structure of type II compactifications with non-trivial Ramond H-fluxes turned on. We focus on the special points in the Calabi-Yau moduli spaces where the H-fluxes are aligned with the vanishing cycles. We will see that the correct identification of the vanishing cycles might be quite subtle, as in the case of the Seiberg-Witten limit. We should note that while our discussion will concentrate on specific Calabi-Yau compactifications, qualitatively the results will be generic, i.e. they are also valid for compactifications on other CY manifolds with the same type of special points in their moduli space.

In chapter 4 we discuss changes of the above scenarios in case NS H-fluxes are turned on. Studying one simple example we see that supersymmetric vacua might be possible away from the special points discussed before.

In the appendix we give additional details about the minimization of the potential in the perturbative heterotic limit with general flux vectors. 
Related issues of H-fluxes in M-theory and type II compactifications on Calabi-Yau fourfolds were discussed in [11] and [12], respectively.

\section{The superpotential from H-fluxes}

\subsection{Special geometry and vector couplings}

The self-couplings of (Abelian) vector multiplets of $\mathcal{N}=2$ supersymmetric Yang-Mills theory are completely specified by a holomorphic function $F(X)$ of the complex scalar components of the $N_{V}$ vector multiplets. With local supersymmetry this function depends on one additional, unphysical scalar field, which incorporates the graviphoton. Including this field, the Abelian gauge group is $G=U(1)^{N_{V}+1}$. The couplings of the vectors are now encoded in a function $F(X)$ of the complex scalars $X^{I}, I=0, \ldots, N_{V}$. $F(X)$ is holomorphic and homogeneous of degree two.

More abstractly, the special geometry [13, 14] of the Kähler manifold $\mathcal{M}$ parameterized by the $N_{V}$ physical scalars is defined in terms of $2\left(N_{V}+1\right)$ covariantly holomorphic sections $L^{I}, M_{I}$ of a bundle $\mathcal{L} \otimes \mathcal{V}$ where $\mathcal{L}$ is a line bundle and $\mathcal{V}$ is a $S p\left(2\left(N_{V}+1\right), \mathbf{Z}\right)$ bundle; i.e. $D_{\bar{A}} L^{I}=\left(\partial_{\bar{A}}-\frac{1}{2} \partial_{\bar{A}} K_{V}(z, \bar{z})\right) L^{I}=0$, and likewise for the $M_{I}$. Here $K_{V}(z, \bar{z})$ is the Kähler potential, and the physical scalars $z^{A}, A=1, \ldots, N_{V}$ are intrinsic complex coordinates on the moduli space $\mathcal{M}_{V}=\mathcal{S K}\left(N_{V}\right)$, which is a special Kähler space of complex dimension $N_{V}$. The sections are assembled into a symplectic vector $V$ :

$$
V=\left(\begin{array}{c}
L^{I} \\
M_{I}
\end{array}\right)
$$

$\mathcal{M}_{V}$ is defined by the constraint

$$
\langle\bar{V}, V\rangle \equiv \bar{V}^{\mathrm{T}} \Omega V=-i
$$

with $\Omega$ the invariant symplectic metric

$$
\Omega=\left(\begin{array}{cc}
0 & 1 \\
-1 & 0
\end{array}\right) .
$$

Given $X^{I}$ and $F_{I}$, one may define an holomorphic period vector $\Xi$,

$$
\Xi(z)=\left(\begin{array}{c}
X^{I}(z) \\
F_{I}(z)
\end{array}\right),
$$

via

$$
X^{I}(z)=e^{-\frac{1}{2} K_{V}(z, \bar{z})} L^{I}, \quad F_{I}(z)=e^{-\frac{1}{2} K_{V}(z, \bar{z})} M_{I}
$$


Via the constraint (2.2) the Kähler potential can be expressed as

$$
K_{V}(z, \bar{z})=-\log \left(i \bar{X}^{I}(\bar{z}) F_{I}(z)-i X^{I}(z) \bar{F}_{I}(\bar{z})\right)=-\log \left(i \Xi^{\dagger} \Omega \Xi\right)
$$

Invariance under $S p\left(2 N_{V}+2\right)$ transformations is manifest.

If $\operatorname{det}\left(\partial_{i} X^{I}, X^{I}\right) \neq 0$, there exists a holomorphic, homogeneous prepotential $F(X)$ such that $F_{I}=\partial F(X) / \partial X^{I}$. In this case the $X^{I}$ are good local homogeneous coordinates on $\mathcal{M}_{V}$; they are algebraically independent, i.e. $\partial_{I} X^{J}=\delta_{I}^{J}$. The existence of a prepotential is a basis-dependent statement. There exists, however, always a symplectic basis $\left(X^{I}, F_{I}\right)$ such that $F_{I}=\partial_{I} F(X)$.

One important example which after a symplectic transformation leads to algebraically dependent periods is given by a prepotential which is linear in one of the sections, say in $X^{1}$ :

$$
F(X)=t^{1} G\left(X^{0}, X^{a}\right)+H\left(X^{0}, X^{a}\right) .
$$

Here we have defined $t^{1}=X^{1} / X^{0}$ and $G, H$ are functions of $X^{0}$ and $X^{a}\left(a=2, \ldots, N_{V}\right)$ only. Then $F_{1}=G / X^{0}$ is independent of $X^{1}$, and after the symplectic transformation $X^{1} \rightarrow \tilde{X}^{1}=F_{1}$ the new $\tilde{X}^{I}$ are algebraically dependent.

If a prepotential exists for the basis $\left(X^{I}, F_{I}\right)$, we can introduce inhomogeneous coordinates $t^{A}$ on $\mathcal{M}_{V}$ which are defined as

$$
t^{A}=\frac{X^{A}}{X^{0}}, \quad X^{0} \neq 0, \quad A=1, \ldots, N_{V}
$$

In this parameterization the Kähler potential is [15]

$$
K_{V}(t, \bar{t})=-\log \left(2(\mathcal{F}+\overline{\mathcal{F}})-\left(t^{A}-\bar{t}^{A}\right)\left(\mathcal{F}_{A}-\overline{\mathcal{F}}_{A}\right)\right)
$$

where $\mathcal{F}(z)=i\left(X^{0}\right)^{-2} F(X)$.

\subsection{Hypermultiplet Couplings and superpotential}

\subsubsection{Gauged $\mathcal{N}=2$ supergravity}

Now consider the $\mathcal{N}=2$ supergravity couplings including $N_{H}$ hypermultiplets $q_{i}$ as additional matter fields [18]. Together with the $N_{V}$ vector multiplets the moduli space is locally, at generic points in the moduli space, a product space of the form

$$
\mathcal{M}=\mathcal{S K}\left(N_{V}\right) \otimes \mathcal{Q}\left(N_{H}\right)
$$

\footnotetext{
${ }^{3}$ Later we will also use inhomogeneous coordinates $T^{A}=-i \frac{X^{A}}{X^{0}}$.
} 
where the hypermultiplet moduli space $\mathcal{M}_{H}=\mathcal{Q}\left(N_{H}\right)$ is a quaternionic space of real dimension $4 N_{H}$. The coupling of the hypermultiplet scalars $q_{i}$ to the vectormultiplets $X^{I}$ arises from gauging the Abelian isometries of $\mathcal{Q}$. This means that the hypermultiplets are charged with respect to the gauge group $G=U(1)^{N_{V}+1}$. The gauging is done by introducing $N_{V}+1$ Killing vectors $k_{I}^{i}(q)$ on $\mathcal{M}_{H}$ which correspond to the (field dependent) Abelian charges of the hypermultiplets. This means that one defines the following covariant derivatives

$$
\nabla_{\mu} q^{i}=\partial_{\mu} q^{i}+k_{I}^{i} A_{\mu}^{I}
$$

The Killing vectors $k_{I}^{i}$ can be determined in terms of a $S U(2)$ triplet of real Killing prepotentials $P_{I}^{x}$ as follows

$$
k_{I}^{i} \Omega_{i j}^{x}=-\left(\partial_{j} P_{I}^{x}+\epsilon^{z y z} \omega_{j}^{y} P_{I}^{z}\right)
$$

where $\omega^{x}$ is a $S U(2)$ connection and $\Omega^{x}$ its curvature.

The gauging of the hypermultiplet isometries generically implies non-vanishing masses of the two $\mathcal{N}=2$ gravitini, whose mass matrix is:

$$
S_{A B}=\frac{i}{2} e^{K_{V} / 2}\left(\sigma_{x}\right)_{A}{ }^{C} \epsilon_{B C} P_{I}^{x}(q) X^{I}(z)=\frac{i}{2}\left(\sigma_{x}\right)_{A}{ }^{C} \epsilon_{B C} P_{I}^{x}(q) L^{I} .
$$

We now rewrite the coupling of the $\mathcal{N}=2$ hypermultiplets to the vector multiplets in $\mathcal{N}=1$ language. The coupling with $x=3$ corresponds to $\mathcal{N}=1$ D-terms while those with $x=1,2$ to F-terms, i.e. they are equivalent to a $\mathcal{N}=1$ superpotential.

From now on we are interested in situations where all $P_{I}^{3}=0$. To derive the superpotential let us introduce the following functions $e_{I}$ :

$$
e_{I}=e^{-K_{H} / 2}\left(P_{I}^{1}+i P_{I}^{2}\right)
$$

Then the $\mathcal{N}=1$ superpotential is

$$
W(z, q)=e_{I}(q) X^{I}(z)
$$

where the $z^{A}$ and also the $q_{i}$ now denote $\mathcal{N}=1$ chiral superfields. We will now motivate (2.15).

The $\mathcal{N}=1$ supergravity action can be expressed in terms of the generalized Kähler function

$$
G=K_{V}(z, \bar{z})+K_{H}(q, \bar{q})+\log |W(z, q)|^{2}
$$

The scalar potential is

$$
v=e^{G}\left(G_{A} G_{\bar{B}} G^{A \bar{B}}+G_{i} G_{\bar{\jmath}} G^{i \bar{\jmath}}-3\right)
$$


and the $\mathcal{N}=1$ gravitino mass

$$
m_{3 / 2}=e^{G / 2}=e^{K / 2}|W|
$$

The introduction of the superpotential eq.(2.15) is largely based on the fact that the mass of the $\mathcal{N}=1$ gravitino in eq.(2.18) agrees with one of the two mass eigenvalues of the two $\mathcal{N}=2$ gravitini in eq.(2.13):

$$
S_{A B}=\frac{i}{2} e^{\left(K_{V}+K_{H}\right) / 2}\left(\begin{array}{cc}
-W+2 i \operatorname{Im}\left(e_{I}\right) X^{I} & 0 \\
0 & W
\end{array}\right)
$$

Indeed, one eigenvalue agrees with (2.18). However for complex $e_{I}$ and $m^{I}$ the mass of the second gravitino is in general different.

\subsubsection{Symplectic covariance}

Since the superpotential eq. 2.15) only contains the periods $X^{I}$ but not the dual periods $F_{I}$, it is clear that the gravitino masses so far are not invariant under symplectic $S p\left(2 N_{V}+\right.$ $2, \mathbf{Z})$ transformations. One can achieve full symplectic invariance by introducing magnetic prepotentials $\tilde{P}^{x I}[2]$. These can be thought of as giving the relevant hypermultiplets also a magnetic charge with respect to the Abelian gauge group $U(1)^{N_{V}+1}$, which can be done by introducing magnetic Killing vectors $\tilde{k}^{i I}$. It then follows that the electric/magnetic prepotentials $\left(P_{I}^{x}, \tilde{P}^{x I}\right)$ as well as the corresponding Killing vectors $\left(k_{I}^{i}, \tilde{k}^{i I}\right)$ transform as vectors under $\operatorname{Sp}\left(2 N_{V}+2\right)$.

In analogy with the $e_{I}$ in eq.(2.14) we introduce the complex magnetic functions

$$
m^{I}=e^{-K_{H} / 2}\left(\tilde{P}^{1 I}+i \tilde{P}^{2 I}\right) .
$$

Then the $e_{I}$ and the $m^{I}$ build a symplectic vector $H$ of the form

$$
H=\left(\begin{array}{c}
m^{I} \\
e_{I}
\end{array}\right)
$$

and the superpotential is given by the symplectic invariant scalar product between $H$ and $\Xi$ :

$$
W(z, q)=\langle H, \Xi\rangle=e_{I}(q) X^{I}(z)-m^{I}(q) F_{I}\left(X^{I}(z)\right),
$$

The $\mathcal{N}=1$ gravitino mass can be simply written as:

$$
m_{3 / 2}=e^{\left(K_{V}+K_{H}\right) / 2}|\langle H, \Xi\rangle|
$$

Finally, the symplectic invariant $\mathcal{N}=2$ gravitino mass matrix is

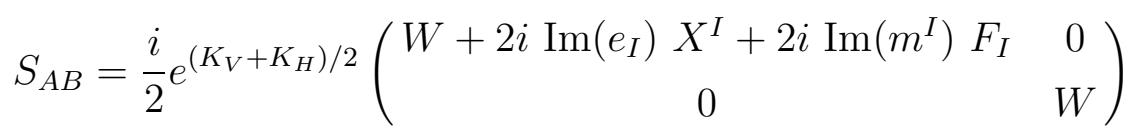


Actually, the symplectic transformations act on the two vectors $\left(P_{I}^{1}, \tilde{P}^{1 I}\right)=$ $e^{K_{H} / 2}\left(\operatorname{Re} e_{I}, \operatorname{Re} m^{I}\right)$ and $\left(P_{I}^{2}, \tilde{P}^{2 I}\right)=e^{K_{H} / 2}\left(\operatorname{Im} e_{I}, \operatorname{Im} m^{I}\right)$. This means that one can always perform symplectic transformations such that, e.g., $\left(P_{I}^{1}, \tilde{P}^{1 I}\right)$ is purely electric. In addition, $\left(P_{I}^{2}, \tilde{P}^{I 2}\right)$ can be also made purely electric by a further symplectic transformations in case these two vectors are local w.r.t. each other, i.e. if

$$
\tilde{P} \times P \equiv P_{I}^{1} \tilde{P}^{2 I}-\tilde{P}^{1 I} P_{I}^{2}=0
$$

If (2.25) holds the superpotential can be always brought to the form eq.(2.15). On the other hand, if $\tilde{P} \times P \neq 0$, i.e. if there exist hypermultiplets with mutually non-local electric/magnetic $U(1)$ charges, the superpotential necessarily contains both $X^{I}$ and $F_{I}$ fields in any basis. Of course, it is not possible to write down a Lorentz invariant microscopic gauged $\mathcal{N}=2$ supergravity action which contains hypermultiplets with mutually non-local electric/magnetic charges. But this case will be of interest for us below, when we investigate points in the Calabi-Yau moduli spaces where electric and dual (magnetic) cycles, which mutually intersect, degenerate (Argyres Douglas points). Here the superpotential will contain both $X^{I}$ and $F_{I}$. We will assume that while a local action without additional auxiliary degrees of freedom does not exist for the nonlocal Argyres-Douglas points, the effective superpotential and the corresponding gravitino mass formulas do provide a valid description for the massless fields.

\subsubsection{The ground state of the theory - the question of partial supersymmetry breaking}

The ground state of the theory is determined by the requirement that the scalar potential is minimized with respect to all scalar fields:

$$
\frac{d v}{d z^{A}}=0, \quad \frac{d v}{d q^{i}}=0 \quad \rightarrow \quad z^{A}=\left.z^{A}\right|_{\min }, \quad q^{i}=\left.q^{i}\right|_{\min } .
$$

$\mathcal{N}=1$ supersymmetry is unbroken at the minimum of the potential if the auxiliary fields are zero

$$
\begin{aligned}
h^{\bar{A}} & =G^{\bar{A} B} e^{G / 2} \partial_{B} G=G^{\bar{A} B}|W| e^{K / 2}\left(\partial_{B} K+\frac{1}{W} \partial_{B} W\right)=0, \\
h^{\bar{\imath}} & =G^{\bar{\imath} j} e^{G / 2} \partial_{j} G=G^{\bar{\imath} j}|W| e^{K / 2}\left(\partial_{j} K+\frac{1}{W} \partial_{j} W\right)=0 .
\end{aligned}
$$

In supergravity, supersymmetric minima of $v$ generally lead to a negative vacuum energy. In order to find minima of $v$ with $\left.v\right|_{\min }=0$ plus unbroken $\mathcal{N}=1$ supersymmetry, all four terms in eqs.(2.27) like $G^{A \bar{B}}|W| e^{K / 2} \partial_{B} K$ etc. must be separately zero. If $G^{\bar{A} B} e^{K / 2} \partial_{B} K$, 
$G^{\bar{\imath} j} e^{K / 2} \partial_{j} K$, and $G^{\bar{A} B} e^{K / 2}, G^{\bar{\imath} j} e^{K / 2}$ are finite this leads to the conditions:

$$
\left.W\right|_{\min }=0,\left.\quad \partial_{A} W\right|_{\min }=0,\left.\quad \partial_{i} W\right|_{\min }=0
$$

If these conditions are satisfied, $\mathcal{N}=2$ supersymmetry is either partially broken to $\mathcal{N}=1$ or unbroken, depending on the eigenvalues of the gravitino mass matrix eq.(2.19). Specifically, if the $e_{I}$ and $m^{I}$ are real at a $\mathcal{N}=1$ minimum, or if $\left.e^{K / 2}\right|_{\min }=0$, then both mass eigenvalues in eq.(2.19) are zero, and the full $\mathcal{N}=2$ supersymmetry will be unbroken.

On the other hand, partial supersymmetry breaking to $\mathcal{N}=1$ supersymmetry is possible if some of the $e_{I}$ or $m^{I}$ are complex. In addition, according to [8], the existence of minima with partial supersymmetry breaking requires that there exists a symplectic basis in which the periods $\tilde{X}^{I}$ are algebraically dependent.

\subsection{The superpotential from type IIB 3-form Fluxes}

\subsubsection{Calabi-Yau compactification}

In type IIB compactifications on a Calabi-Yau threefold $M$ the superpotential eq.(2.15) arises from turning on flux for NS and R three-form field strength $H_{N S}^{(3)}$ and $H_{R}^{(3)}$ [3]. The low energy spectrum consists, in addition to the $\mathcal{N}=2$ gravitational multiplet with the graviphoton, of $N_{V}=h^{2,1}$ vector multiplets and $N_{H}=h^{1,1}+1$ hypermultiplets. Turning on the internal H-flux manifests itself in the 4-dimensional effective Lagrangian as a superpotential of the form

$$
W=\int \Omega \wedge\left(\tau H_{N S}^{(3)}+H_{R}^{(3)}\right)
$$

where $\Omega$ is the holomorphic 3 -form on the Calabi-Yau space and $\tau$ the complex type IIB couplings constant. This superpotential is closely related to NS5 resp. D5 branes wrapped around 3-cycles $\mathcal{C}^{(3)}$ in the Calabi-Yau space. In the four-dimensional effective theory the wrapped 5-branes correspond to domain walls whose BPS tension is the jump $\Delta W$ of the superpotential across the wall.

In order to bring eq.(2.29) to the form (2.15) one expands the 3-cycles dual to the H-fluxes in terms of the basis vectors $\left(A^{I}, B_{I}\right)$ of $H_{3}(M, \mathbf{Z})$ as

$$
\tau \mathcal{C}_{N S}^{(3)}+\mathcal{C}_{R}^{(3)}=e_{I}(\tau) A^{I}-m^{I}(\tau) B_{I}
$$

where $e_{I}(\tau)$ and $m^{I}(\tau)$ are defined as

$$
e_{I}(\tau)=e_{I}^{1} \tau+e_{I}^{2}, \quad m^{I}(\tau)=m^{1 I} \tau+m^{2 I} .
$$


The integer symplectic vectors $\left(e_{I}^{1}, m^{1 I}\right)$ and $\left(e_{I}^{2}, m^{2 I}\right)$ are the quantized flux values of the NS resp. R 3-form fields through the 3-cycles. Then the superpotential (2.29) becomes 3]

$$
\begin{aligned}
W=\int_{\mathcal{C}_{R}^{(3)}} \Omega+\tau \int_{\mathcal{C}_{N S}^{(3)}} \Omega=W_{R}+\tau W_{N S}=e_{I}(\tau) X^{I}-m^{I}(\tau) F_{I} \\
W_{R}=e_{I}^{2} X^{I}-m^{2 I} F_{I} \\
W_{N S}=e_{I}^{1} X^{I}-m^{1 I} F_{I}
\end{aligned}
$$

here $X^{I}=\int_{A^{I}} \Omega$ and $F_{I}=\int_{B_{I}} \Omega$. Similar superpotentials were already discussed in [6]. This superpotential $W$ is $S p\left(2 N_{V}+2, \mathbf{Z}\right)$ invariant. Under the type IIB S-duality transformations $\tau \rightarrow \frac{a \tau+b}{c \tau+d}$ it transforms with modular weight -1 ,

$$
W \rightarrow \frac{W}{c \tau+d}
$$

provided that $\left(H_{N S}^{(3)}, H_{R}^{(3)}\right)$, or equivalently $\left(e_{I}^{1}, e_{I}^{2}\right)$ and $\left(m^{1 I}, m^{2 I}\right)$ transform as vectors under $S L(2, \mathbf{Z})$.

\subsubsection{The scalar potential and the question of partial supersymmetry break- ing}

Next we have to determine the Kähler potential and the scalar potential, which receives contributions from the scalar fields in both, vector and hypermultiplets. On the hypermultiplet side we are dealing with the complex dilaton field $\tau$ and $Y=(\operatorname{vol}(\mathrm{CY}))^{1 / 3}+i a$, the volume of the Calabi-Yau space and its axionic partner $a$, plus possibly other complex fields $q_{i}$. We assume that the vacuum expectation values of the $q_{i}$ are zero and thus we can neglect them in the following discussion; we are mainly interested in the contributions of $\tau$ and $Y$ to the Kähler potential. We work from now on in the weak coupling limit, i.e. $\tau \rightarrow \infty$ and in the large volume limit, i.e. $Y \rightarrow \infty$. In these two limits the Kähler potential is explicitly known :

$$
K=K_{V}+K_{H}, \quad K_{H}=-\log \left(\frac{1}{2 i}(\tau-\bar{\tau})\right)-3 \log (Y+\bar{Y}) .
$$

The function $G=K+\log |W|^{2}$ is invariant under $S L(2, \mathbf{Z})_{\tau}$.

Since the superpotential (2.32) does not depend on the field $Y$, the contribution of $Y$ to the scalar potential $v$ has precisely the effect to cancel the negative vacuum energy. Specifically, the scalar potential now is

$$
v=e^{G}\left(G_{A} G_{\bar{A}} G^{A \bar{B}}+G_{\tau} G_{\bar{\tau}} G^{\tau \bar{\tau}}\right) .
$$


Supersymmetric minima of $v$ require that the auxiliary fields $h$ Since the superpotential does not depend on $Y$ and on the other hypermultiplets $q_{i}$, the conditions $D_{Y} W=D_{q_{i}} W=0$ imply $W=0$, and therefore the conditions of unbroken local $\mathcal{N}=1$ supersymmetry turn into the conditions of unbroken global $\mathcal{N}=1$ supersymmetry, which read:

$$
\frac{d W}{d z^{A}}=0, \quad \frac{d W}{d \tau}=0, \quad W=0 .
$$

Using the specific form eq.(2.32) of $W$ these conditions turn into

$$
W_{N S}=0, \quad W_{R}=0, \quad \frac{d W_{R}}{d z^{A}}+\tau \frac{d W_{N S}}{d z^{A}}=0 .
$$

Let us now consider the question of partial supersymmetry breaking and compute the gravitino mass matrix (2.19) for the H-flux induced superpotential (2.32). With $(2.32,2.34)$ the gravitino mass matrix (2.24) becomes

$$
S_{A B}=\frac{i}{2(\tau-\bar{\tau})^{1 / 2}(Y+\bar{Y})^{3 / 2}} e^{K_{V} / 2}\left(\begin{array}{cc}
-W+2 i \operatorname{Im} \tau W_{N S} & 0 \\
0 & W
\end{array}\right) .
$$

We see that a priori partial supersymmetry breaking (one vanishing eigenvalue) is only possible in the presence of NS fluxes and $\operatorname{Im}(\tau) \neq 0$. However if we treat $\tau$ as a dynamical field we have to require that $d W / d \tau=0$ and hence $W_{N S}=0$. Therefore, as soon as we are searching for $\mathcal{N}=1$ supersymmetric vacua, with $d W / d \tau=0$, both gravitino eigenvalues are zero and the theory is $\mathcal{N}=2$ supersymmetric. In other words, partial supersymmetry breaking seems to be impossible in connection with the H-flux induced superpotential eq.(2.32); supersymmetry is either completely broken, or supersymmetric minima always preserve full $\mathcal{N}=2$ supersymmetry.

\subsubsection{The type IIB superpotential from gauged $\mathcal{N}=2$ supergravity}

Comparing the superpotential eq.(2.32) with the general expressions in the previous section, we can derive the following electric and magnetic Killing prepotentials for the hypermultiplet fields $\left(\tau=\tau_{1}+i \tau_{2}\right)$ :

$$
\begin{aligned}
P_{I}^{1} & =e^{K_{H} / 2} \operatorname{Im} e_{I}=\frac{\tau_{2}^{1 / 2}}{(Y+\bar{Y})^{3 / 2}} e_{I}^{1}, \\
P_{I}^{2} & =e^{K_{H} / 2} \operatorname{Re} e_{I}=\frac{1}{\tau_{2}^{1 / 2}(Y+\bar{Y})^{3 / 2}}\left(\tau_{1} e_{I}^{1}-e_{I}^{2}\right), \\
\tilde{P}^{1 I} & =e^{K_{H} / 2} \operatorname{Im} m^{I}=\frac{\tau_{2}^{1 / 2}}{(Y+\bar{Y})^{3 / 2}} m^{1 I}, \\
\tilde{P}^{2 I} & =e^{K_{H} / 2} \operatorname{Re} m^{I}=\frac{1}{\tau_{2}^{1 / 2}(Y+\bar{Y})^{3 / 2}}\left(\tau_{1} m^{1 I}-m^{2 I}\right) .
\end{aligned}
$$


Similar as in [2] these prepotentials should be obtained from the electric resp. magnetic gauging of the hypermultiplets $Y$ and $q=\left(S, C_{0}\right)$, where the complex dilaton field $S$ is the NS component of $q$, and $C_{0}$ its complex Ramond component. Gauging in an $S L(2, \mathbf{Z})$ invariant way two particular isometries of the hypermultiplet moduli space $\mathcal{M}_{H}=S U(2,1) / S U(2) \times U(1)$ will lead to the Killing prepotential (2.39) $)^{\text {f. Note that }}$ the condition that the electric and magnetic charges are mutually local is equivalent to the locality of the Ramond and NS flux vectors, i.e.

$$
\int H_{N S}^{(3)} \wedge H_{R}^{(3)} \sim m \times e=m^{1 I} e_{I}^{2}-m^{2 I} e_{I}^{1}=0 .
$$

As we will discuss in the last section, some special H-fluxes which do not satisfy this constraint can also lead to supersymmetric vacua.

\subsection{The superpotential from type IIA fluxes}

Consider now type IIA compactification on the mirror Calabi-Yau space $W$ with Hodge numbers $h^{2,1}(W)=h^{1,1}(M)$ and $h^{1,1}(W)=h^{2,1}(M)$. The number of vectormultiplets is $N_{V}=h^{1,1}(W)$, and the number of hypermultiplets is $N_{H}=h^{2,1}(W)+1$. The type IIA superpotential can be obtained performing the mirror map on the type IIA superpotential eq.(2.32). Since the IIA mirror configuration to the wrapped IIB NS 5-branes is unknown, we discuss the case of turning on Ramond fluxes only. The mirror flux of $H_{R}^{(3)}$ corresponds to fluxes of the IIA Ramond fields $H_{R}^{(6)}, H_{R}^{(4)}$ and $H_{R}^{(2)}$ which are dual to 0,2 and 4-cycles on $W$, plus one other flux term corresponding to the 6-cycle, $W$ itself.

We will define the IIA flux vectors with respect to the integral basis $\Xi_{\infty}$ (see sect. 3.1). Then the IIA superpotential is [3]

$$
\begin{aligned}
W & =\int_{W}\left(H_{R}^{(6)}+J \wedge H_{R}^{(4)}+J \wedge J \wedge H_{R}^{(2)}+m^{0} J \wedge J \wedge J\right)= \\
& =e_{0}+\int_{\mathcal{C}_{R}^{(2)}} J+\int_{\mathcal{C}_{R}^{(4)}} J \wedge J+m^{0} \int_{W} J \wedge J \wedge J,
\end{aligned}
$$

where $J$ is the Kähler class of $W$. The corresponding domain walls are due to D2branes, living in the uncompactified space, D4-branes wrapped around the 2-cycles $\mathcal{C}_{R}^{(2)}$, D6-branes wrapped around $\mathcal{C}_{R}^{(4)}$ and D8-branes wrapped around the entire CY-space $W$. Next we choose a basis $J^{A}\left(A=1, \ldots h^{1,1}\right)$ for $H_{2}(W, \mathbf{Z})$,

$$
\mathcal{C}_{R}^{(2)}=e_{A} J^{A}
$$

as well as a dual basis $\tilde{J}_{A}$ for $H_{4}(W, \mathbf{Z})\left(J_{A} \wedge \tilde{J}_{A}=\Omega \wedge \bar{\Omega}\right.$, no sum on $\left.A\right)$

$$
\mathcal{C}_{R}^{(4)}=m^{A} \tilde{J}_{A}
$$

\footnotetext{
${ }^{4}$ We acknowledge discussions with G. Dall'Agata and J. Louis.
} 
The integers $e_{A}$ and $m^{A}$ are the quantized fluxes of $H_{R}^{(4)}$ and $H_{R}^{(2)}$ through the 4- and 2-cycles, respectively. Then the type IIA superpotential (2.41) can be written in homogeneous coordinates as

$$
W=e_{I} X^{I}-m^{I} F_{I},
$$

where we have replaced the $e_{0}$ by $e_{0} X^{0}$. Therefore the classical IIA periods $X^{0}, X^{A}$ are associated with the 0 - and 2-cycles of $W$, whereas the periods $F_{A}$ and $F_{0}$ correspond to the 4 - and 6-cycles. The integers $\left(e_{I}, m^{I}\right)$ transform as a vector under $S p\left(2 h^{1,1}(W)+2, \mathbf{Z}\right)$.

\subsection{Relation between the superpotential due to Ramond fluxes and $\mathcal{N}=2$ black holes and attractor mechanism}

The above discussion of the superpotential due to internal fluxes has a close relationship to extremal black hole solutions in $\mathcal{N}=2$ supergravity, which we will now exhibit. We will show that the supersymmetry condition $h^{\bar{A}}=0$ (see eq.(2.27)) is formally analogous to the attractor equations which determines the values of the scalar fields at the horizon of $\mathcal{N}=2$ supersymmetric black holes.

Consider $\mathcal{N}=2$ BPS states, whose masses are equal to the central charge $Z$ of the $\mathcal{N}=2$ supersymmetry algebra. The magnetic/electric charge vector of the BPS states is defined as

$$
\begin{aligned}
& p^{I}=\frac{1}{2 \pi} \int_{S^{2}} F^{I}, \\
& q_{J}=\frac{1}{2 \pi} \int_{S^{2}} G_{J},
\end{aligned}
$$

where $F^{I}$ and $G_{J}$ are the electric and magnetic Abelian field strengths in four dimensions. In terms of the charge vector $Q=\left(p^{I}, q_{I}\right)$ and the period vector $V$ the BPS masses are [19]:

$$
M_{B P S}^{2}=|Z|^{2}=|\langle Q, V\rangle|^{2}=e^{K_{V}}\left|q_{I} X^{I}(z)-p^{I} F_{I}(z)\right|^{2} \equiv e^{K_{V}}|\mathcal{M}(z)|^{2} .
$$

Extremal $\mathcal{N}=2$ black holes solution leave half of the supersymmetries unbroken. They are BPS states. In type II string theory they can be constructed as D-branes wrapped around the internal CY cycles, where the wrapping numbers corresponds to the electric and magnetic charges. Specifically in type IIB, the black holes arise from wrapped D3branes around 3-cycles, whereas in type IIA black holes originate from wrapping D6, D4, D2 and D0-branes over the cycles of the respective dimensions.

Near the horizon the values of the moduli fields, and thus the value of the central charge, are strongly restricted by the presence of full $\mathcal{N}=2$ supersymmetry. In [9] it was 
proved that this implies that the central charge becomes extremal on the horizon. As a consequence, independent of their asymptotic values, at the horizon the moduli are uniquely determined in terms of the magnetic/electric charges $p^{I}$ and $q_{I}$. This is called the attractor mechanism. The value of the central charge at the horizon is related to the Hawking-Bekenstein entropy via

$$
\frac{\mathcal{S}}{\pi}=\left|Z_{\mathrm{hor}}\right|^{2}
$$

In order to obtain the attractor values of the moduli at the horizon for extremal $\mathcal{N}=2$ black holes, one has to determine the extremal value of the central charge in moduli space. This implies

$$
\partial_{A}|Z|=0 \quad \leftrightarrow \quad D_{A} \mathcal{M}=0
$$

These equations are difficult to solve in general. They are, however, equivalent to the following set of algebraic equations [9]

$$
\bar{Z} V-Z \bar{V}=i Q
$$

Several solutions of these equations in the context of Calabi-Yau black holes were discussed in 20, 21].

Comparing the extremal black holes with the $\mathcal{N}=1$ supergravity action we get the following formal correspondence between the BPS masses of the $\mathcal{N}=2$ black holes and the $\mathcal{N}=1$ superpotential,

$$
\mathcal{M} \cong W, \quad \text { with } q \cong e, \quad p \cong m
$$

as well as the correspondence between the black hole entropy and the gravitino masses,

$$
\frac{\mathcal{S}}{\pi} e^{K_{H}} \cong m_{3 / 2}^{2}
$$

The extremization of the central charges at the horizon, eqs.(2.48) and (2.49), corresponds to the condition of vanishing auxiliary fields $D_{A} W=0$, i.e. to unbroken $\mathcal{N}=1$ supersymmetry. The condition $m_{3 / 2}^{2}=0$ is equivalent to dealing with an extremal black hole with vanishing entropy. Therefore the supersymmetric points of the effective supergravity action precisely correspond to the attractor points of the $\mathcal{N}=2$ black holes. These observations will turn out to be useful to find explicitly the points of preserved $\mathcal{N}=1$ supersymmetry, since the equations $D_{A} W=0$ can be translated into the following equation for the symplectic vectors $V$ and $H$ :

$$
e^{K_{V} / 2}(\bar{W} V-W \bar{V})=i H
$$




\section{Type II Vacua with Ramond Fluxes}

In this section we will consider type IIB compactifications with all NS 3-form fields turned off. Then the superpotential (2.22) does not depend on the scalar fields of the universal hypermultiplet. It also means that the fluxes are mutually local. The condition of having unbroken supersymmetry at the minimum of the scalar potential then has solutions only at subsets of the boundary of the moduli space [3, 4].

\subsection{Type II compactifications on Calabi-Yau threefolds}

Let us review the aspects of the geometry which will be relevant for the discussion of fluxes and the question of supersymmetry breaking. The vector moduli space is completely geometrical in the type IIB compactification on Calabi-Yau threefolds $M$. This special Kähler manifold arises as the moduli space of complex structure deformations of $M$ for the type IIB string compactification on $M$. By mirror symmetry, it is equivalent to the complexified Kähler structure deformation space on the mirror $W$ of $M$, which describes the type IIA string vector moduli space on $W$.

Let us now investigate the basis of the fluxes in type IIA/B compactifications. In type IIB compactifications on $M$ the fluxes of the 3 -form field strengths $H_{R}^{(3)}$ and $H_{N S}^{(3)}$ are w.r.t. an integral symplectic basis of $H^{3}(M)$. Following [23, 24] we will find such an integral symplectic basis for the periods, or equivalently a basis for $H_{3}(M, \mathbf{Z})$ at the point of maximal unipotent monodromy, which corresponds in the mirror $W$ to the large volume limit $\left(R^{A}\right)^{2} \rightarrow \infty$. At this point, which is at $z^{A}=0$ in the coordinates used in [24], one has a unique analytic period, normalized to $X^{0}=1+\mathcal{O}(z)$, and $m=\operatorname{dim}\left(H^{1}(M, \Theta)\right)=h^{2,1}(M)$ logarithmic periods $X^{A}$, which provide natural special Kähler coordinates $t^{A}=\frac{X^{A}}{X^{0}}=\frac{1}{2 \pi i} \log \left(z^{A}\right)+\sigma_{A}$, where $\sigma_{A}=\mathcal{O}(z)$ and $t^{A}:=B^{A}+i\left(R^{A}\right)^{2}$. The prepotential $F$, which is homogeneous of degree two in the periods $X^{I}$, is $\left(q_{A}=\right.$ $\left.\exp \left(2 \pi i t^{A}\right)\right)$

$$
\begin{aligned}
F & =-\frac{C_{A B C} X^{A} X^{B} X^{C}}{3 ! X^{0}}+n_{A B} \frac{X^{A} X^{B}}{2}+c_{A} X^{A} X^{0}-i \frac{\chi \zeta(3)}{2(2 \pi)^{3}}\left(X^{0}\right)^{2}+\left(X^{0}\right)^{2} f(q) \\
& =\left(X^{0}\right)^{2} \mathcal{F}=X_{0}^{2}\left[-\frac{C_{A B C} t^{A} t^{B} t^{C}}{3 !}+n_{A B} \frac{t^{A} t^{B}}{2}+c_{A} t^{A}-i \frac{\chi \zeta(3)}{2(2 \pi)^{3}}+f(q)\right]
\end{aligned}
$$

It defines an integral basis for the periods in the following way (note that in the following

\footnotetext{
${ }^{5}$ For a review on string vacua with $\mathcal{N}=2$ supersymmetry see 22
} 
the periods $F_{I}$ are ordered in a different way compared to eq.(2.4))

$$
\Xi_{\infty}=\left(\begin{array}{c}
X^{0} \\
X^{A} \\
\frac{\partial F}{\partial X^{A}} \\
\frac{\partial F}{\partial X^{0}}
\end{array}\right)=X_{0}\left(\begin{array}{c}
1 \\
t^{A} \\
\frac{\partial \mathcal{F}}{\partial t^{A}} \\
2 \mathcal{F}-t^{A} \partial_{A} \mathcal{F}
\end{array}\right)=X^{0}\left(\begin{array}{c}
1 \\
t^{A} \\
-\frac{C_{A B C}}{2} t^{B} t^{C}+n_{A B} t^{B}+c_{A}+\partial_{A} f(q) \\
\frac{C_{A B C}}{3 !} t^{A} t^{B} t^{C}+c_{A} t^{A}-i \frac{\chi \zeta(3)}{(2 \pi)^{3}}+\mathcal{O}(q)
\end{array}\right) .
$$

In the type IIA interpretation $C_{A B C}=\int_{W} J_{A} J_{B} J_{C} \geq 0$ are the classical intersection numbers, where $J_{A}$ are $(1,1)$-forms in $H^{2}(W, \mathbf{Z})$, which span the Kählercone, $c_{A}=\frac{1}{24} \int_{W} c_{2} J_{A} \bar{\rrbracket}$. In type IIA the $q$ expansion of $F$ around the large volume is a world sheet instanton expansion. The explicit form $f(q)$ can be determined by mirror symmetry using the type IIB compactification on $M$.

Note that the point $q_{A}=0, \forall A$ corresponds, by mirror symmetry, to a very singular configuration of $M$ (it degenerates to intersecting hyperplanes), i.e. from the Type IIB perspective the large volume limit of $W$ corresponds to a complex structure degeneration of $M$, where partial susy breaking might occur. Away from this point, in a generic direction in the complex structure moduli space, $M$ is regular and we do not expect any unbroken supersymmetry. Going away from the supersymmetric groundstate world-sheet instantons on the mirror $W$ will break supersymmetry [4], but the dynamics generically drives the theory back to its supersymmetric vacuum.

Interesting effects may also occur at other singular points on the moduli space of $M$, like the conifold points. Here one particular IIB 3 -cycle $A^{1}$ shrinks to zero size, i.e. $X_{\mathrm{IIB}}^{1} \rightarrow 0$. However that does not correspond to a shrinking type IIA 2-cycle. Rather the whole quantum volume of $W$, i.e. the period $F_{0}$, vanishes [1]. In the next section we will discuss this and other degeneration points in the Calabi-Yau moduli space.

In the type IIA interpretation of the period vector $\Xi, t^{A}$ scales as the third root of the volume of the threefold $W$. This relates via (2.46) the first entry of (3.2) to the $D 0$-mass, the next $m$ entries to the BPS masses of the wrapped $D 2$-branes, followed by the masses of the $m D 4$ and finally the last entry to the $D 6$-brane wrapped around the whole CalabiYau manifold $W$. This identification of the basis of $H^{3}(M, \mathbf{Z})$ and $\bigoplus_{i=0}^{3} H^{i, i}(W, \mathbf{Z})$ maps

\footnotetext{
${ }^{6}$ This basis is unique up to integral symplectic transformations. E.g. a slightly more complicated choice has been made in [23], which amounts to a shift of the $C_{A}$ by an integer. Odd $C_{A B C}$ requires that some of the $n_{A B} \in \mathbf{Z} \backslash\{0\}$ to get an integer monodromy around $z_{A}=0$. Using mirror symmetry and the expression for the $D 4$-brane charge one can determine $n_{A B}$ as the integral of $J_{A} \wedge J_{B}$ against $i_{*} c_{1}(D)(i: D \hookrightarrow W)$ where $D$ is the divisor dual to $J_{A} \wedge J_{B}$ [40, 41, 42].

${ }^{7}$ Note that, up to the $n_{A B}$, the data needed to specify (3.2) are those which give the topological classification of the three-fold $W$ as it follows from a theorem of C.T.C. Wall.

${ }^{8}$ The moduli space of the $D 0$-brane, $W$ itself, has been identified with the moduli space of the $D 3$ -
} 
a IIB RR 3-form $H_{R}^{(3)}$ to a linear combination in $\oplus_{i=0}^{3} H_{R}^{i, i}$ of type IIA RR forms.

\subsection{Points in the moduli space corresponding to a nonsingular CY}

In the absence of $H^{N S}$, all cycles can be chosen to be $A$-cycles, say $A_{1}$ and $A_{2}$. It is assumed that the periods $X^{I}=\int_{A_{I}} \Omega$ can serve as homogeneous coordinates in the moduli space, in particular that they are algebraically independent, $\frac{\partial X^{I}}{\partial X^{J}}=\delta_{J}^{I}$. If $e^{K} G^{A \bar{B}}$ (cf. eq.(2.27) ) is finite at the point in the moduli space under consideration then the condition for unbroken supersymmetry is $W=0$ and $D_{A} W=\left(\partial_{A}+K_{A}\right) W=0 \forall A$. This is equivalent to $W=\mathrm{d} W=0$. Here the derivatives are w.r.t. the inhomogeneous coordinates $t^{A}=\frac{X^{A}}{X^{0}}$. For a superpotential of the form 2.32) this is equivalent to $\mathrm{d} W=\frac{\partial W}{\partial X^{I}} \mathrm{~d} X^{I}=0$ and requires that $H^{R}=0$.

When could $X$ fail to be a suitable parametrization for the complex structure moduli space? In fact this happens even at points parametrizing non-singular Calabi-Yau manifolds. Let us consider for example the mirror of the sextic $W=2 x_{0}^{3}+\sum_{i=1}^{4} x_{i}^{6}-\psi \prod_{i=0}^{4} x_{i}=$ 0 in $\mathbf{P}(2,1,1,1,1)$ discussed in [17, 10]. With the data $\chi=-204, C_{A A A}=3$ and $\int c_{2} J=42$ we find an integral basis $\Xi_{\infty}$ at the point of maximal unipotent monodromy $z=\frac{1}{(6 \psi)^{6}}=0$ from (3.1,3.2). This can be analytically continued to $\psi=0$. Here we find a basis of solutions $\Xi_{0}=\left(w_{2}, w_{1}, w_{0}, w_{5}\right)$ with

$$
w_{0}=-\frac{i \pi^{4}}{3^{5}} \sum_{n=1}^{\infty} \frac{e^{\frac{5 n i \pi}{6}}}{\sin \frac{\pi n}{6} \Gamma(n) \Gamma^{4}\left(1-\frac{n}{3}\right) \Gamma^{4}\left(1-\frac{n}{6}\right)}\left(\frac{6 \psi}{2^{\frac{1}{3}}}\right)^{n}, \quad w_{k}=w_{0}\left(e^{\frac{2 \pi i k}{6}} \psi\right)
$$

The transformation matrix $\Xi_{\infty}=N \Xi_{0}$ is

$$
N=\left(\begin{array}{rrrr}
-1 & -1 & 1 & 1 \\
0 & 0 & -3 & 0 \\
0 & -3 & 3 & 0 \\
3 & 0 & -9 & -6
\end{array}\right)
$$

It follows from this that $X=6 X_{1}+3 F_{1}-9 X_{0}-2 F_{0}=c \psi^{2}+\mathcal{O}\left(\psi^{3}\right)$ Is not a good coordinate for the moduli space and with $W=X, W=\mathrm{d} W=0$ can be fulfilled. However, we see that due to the degeneration of the factor $e^{K} \sim \frac{1}{|\psi|^{2}}$ (the metric stays finite) the scalar potential does not vanish"ण

$$
V=e^{K} G^{\psi \bar{\psi}}\left|D_{\psi} W\right|^{2}=\tilde{c}+\mathcal{O}\left(|\psi|^{2}\right),
$$

brane on the special Lagrangian torus (in $M$ ) fibered over the $S^{3}$, which vanishes at the generic conifold [26.

$$
\begin{aligned}
{ }^{9} c & =\frac{i \pi^{4} 2^{10 / 3}}{9 \sqrt{3} \Gamma\left(\frac{1}{3}\right) \Gamma\left(\frac{2}{3}\right)} \\
{ }^{10} \tilde{c} & =\frac{8 \pi^{2}}{177147 \sqrt{3} \Gamma^{2}\left(\frac{1}{3}\right) \Gamma^{6}\left(\frac{2}{3}\right) \Gamma^{16}\left(\frac{5}{6}\right)}
\end{aligned}
$$


and supersymmetry is broken.

The periods at a generic point $\psi_{0}$ of the moduli space are all power series in the deformation parameter $\tilde{a}=\psi-\psi_{0}$ of the Calabi-Yau space. For simplicity consider a one parameter family $h^{11}=1$ and with Ramond fluxes only. We may choose a new variable $a$ as a fractional power of $\tilde{a}$ so that only integer powers of $a$ appear in the periods. Let us be concrete and consider the generic expansion of the periods around the point $a=0$

$$
\Xi_{k}=\sum_{n=0}^{\infty} c_{k, n} a^{n}
$$

where generically all coefficients $c_{k, n}$ are non-zero. Iff at the point $a=0$ the first two coefficients $c_{k, n}, n=0,1$, of the periods would be linear dependent over the rational numbers, then we could pick a flux whose dual $X^{A}$ is not a good variable of the moduli space at $a=0$, i.e. $X \sim a^{2}+\mathcal{O}\left(a^{3}\right)$. More precisely, if $X=x_{k} \Xi_{k}$, the $x_{k}$ have to satisfy

$$
\sum_{k=1}^{2 h_{11}+2} x_{k} c_{k, n}=0, \quad n=0,1
$$

For the statement that all choices of $A$-cycles lead to good algebraically independent coordinates to hold the ratios $\frac{x_{k}}{x_{l}}$ should be irrational for all $k, l$. Clearly the above equation can be solved for $x_{k} \in \mathbf{C}$. An interesting question is whether it can be solved for $x_{k} \in \mathbf{R}$. In this case it would be possible to select fluxes with "large" integer coefficients which would lead with an arbitrary precision to a supersymmetric vacuum at the point $a=0$. To check this we plotted

$$
\operatorname{det}\left(\begin{array}{cc}
\operatorname{Re}\left(c_{0}^{1}\right) & \ldots \operatorname{Re}\left(c_{0}^{4}\right) \\
\operatorname{Im}\left(c_{0}^{1}\right) & \ldots \operatorname{Im}\left(c_{0}^{4}\right) \\
\operatorname{Re}\left(c_{1}^{1}\right) & \ldots \operatorname{Re}\left(c_{1}^{4}\right) \\
\operatorname{Im}\left(c_{1}^{1}\right) & \ldots \operatorname{Im}\left(c_{1}^{4}\right)
\end{array}\right)
$$

for the quintic hypersurface in $\mathbf{P}^{4}$ and found that is vanishes only at the orbifold point, which implies that there is not even approximate supersymmetry for any choice of RR fluxed for a generic quintic.

\subsection{Overview over the degenerate cases}

As already emphasized, supersymmetry will be broken at generic points in the CalabiYau moduli space. However there is in fact the chance that supersymmetric minima exist at those points where the Calabi-Yau space degenerates. These points correspond

\footnotetext{
${ }^{11}$ The infinitesimal Torelli Theorem implies only that there is one choice of $h_{2,1}+1$ cycles, whose periods can serve as good homogeneous parameters.
} 
to limits where certain cycles of the Calabi-Yau space shrink to zero size (resp. grow to infinity). As we will see these degenerate points will correspond to supersymmetric vacua in case the flux vectors are precisely aligned along the directions of the vanishing cycles.

Let us analyze the situation in more detail. Suppose that we are considering a superpotential of the form $W=e_{1} X^{1}$, where e.g. in type IIA, $X^{1}$ corresponds to a two-cycle, $X^{1}=\int_{\mathcal{C}_{1}^{(2)}} J$ and the flux is due to a non-vanishing four-form $H_{R}^{(4)}: e_{1}=\int_{\mathcal{C}_{1}^{(4)}} H_{R}^{(4)}$. Then, at a generic point in the moduli space, where $X^{1} \neq 0$, the condition $W=d W=0$ implies that $H_{R}^{(4)}=0$, i.e. non-vanishing flux necessarily breaks supersymmetry. On the other hand, in case the two-cycle vanishes, $X^{1} \rightarrow 0$, the condition $W=0$ is automatically satisfied. Moreover the metric factor $e^{K} G^{1 \overline{1}}$ vanishes in many examples at the points where $X^{1}=0$. Hence (2.27) is also satisfied. We will show in the following that the degeneration points also correspond to minima of the scalar potential, which means that they are supersymmetric ground states of the theory. As mentioned already, the values of the scalar fields at these points precisely agree with the attractor points in the context of supersymmetric black holes. So it is quite natural to assume that the compactification is dynamically driven to the attractor points in case we turn on H-fluxes which are aligned along vanishing cycles.

Before we proceed let us first give a brief overview over the degenerate cases with vanishing cycles in the IIA moduli space and the corresponding Ramond fluxes which are turned on. For simplicity consider for the moment a model with $h^{1,1}=2$; the corresponding two moduli are $S=-i X^{1} / X^{0}$ and $T=-i X^{2} / X^{0}$. In the large volume $\operatorname{Re} S, \operatorname{Re} T \gg 1$ and $\operatorname{Re} S>\operatorname{Re} T$ limit, where $F \sim i S T^{2}$, we have the following correspondences (we assume that the CY is a K3 fibration over a $\mathbf{P}_{b}^{1}$ base; the K3 fiber contains a second $\mathbf{P}^{1}$, denoted by $\left.\mathbf{P}_{f}^{1}\right)$ :

$$
\begin{aligned}
X^{0} & \Longleftrightarrow \operatorname{vol}\left(\mathcal{C}^{(0)}\right), \\
X^{1} \sim i S & \Longleftrightarrow \operatorname{vol}\left(\mathcal{C}_{1}^{(2)}\right) \sim \operatorname{vol}\left(\mathbf{P}_{b}^{1}\right), \\
X^{2} \sim i T & \Longleftrightarrow \operatorname{vol}\left(\mathcal{C}_{2}^{(2)}\right) \sim \operatorname{vol}\left(\mathbf{P}_{f}^{1}\right), \\
F_{1} \sim i \frac{\partial F}{\partial S} \sim T^{2} & \Longleftrightarrow \operatorname{vol}\left(\mathcal{C}_{1}^{(4)}\right) \sim \operatorname{vol}(K 3), \\
F_{2} \sim i \frac{\partial F}{\partial T} \sim S T & \Longleftrightarrow \operatorname{vol}\left(\mathcal{C}_{2}^{(4)}\right), \\
F_{0} \sim i S T^{2} & \Longleftrightarrow \operatorname{vol}\left(\mathcal{C}^{(6)}\right) \sim \operatorname{vol}(C Y) .
\end{aligned}
$$

Here $\mathcal{C}^{(d)}$ is a cycle of real dimension $d . \operatorname{vol}\left(\mathcal{C}^{(0)}\right)$ is a constant and $\mathcal{C}_{2}^{(4)}$ is a four-cycle which contains the base $\mathbf{P}_{b}^{1}$ and the $\mathbf{P}_{f}^{1}$. All volumes are meant to be complexified volumes. In 
the following the aligned fluxes will correspond to the vanishing cycles. However other, non-aligned choices are of course also possible and will be mentioned in the sects. 3.4-3.9.

\section{(i) The perturbative heterotic limit}

This is simply the limit where, in the heterotic dual, we turn off all instanton effects, i.e. $S \rightarrow \infty$. Therefore, comparing with (3.9) we see that the cycles $\mathcal{C}_{1}^{(2)}, \mathcal{C}_{2}^{(4)}, \mathcal{C}^{(6)}$ become large, which can be alternatively interpreted to mean that the remaining cycles $\mathcal{C}^{(0)}, \mathcal{C}_{2}^{(2)}$ and $\mathcal{C}_{1}^{(4)}$ vanish. Turning on the aligned fluxes $e_{0}, e_{2}$ and $m^{1}$, the superpotential takes the form

$$
W=e_{0} X^{0}+e_{2} X^{2}+m^{1} F_{1}
$$

Note that the periods $X^{2}$ and $F_{1}$ (and $X^{0}=1$ anyway) do not vanish. The superpotential is zero at the minimum, and $\mathrm{d} W=0$, nevertheless supersymmetry will be unbroken because of the $e^{K}$ factor, cf. the discussion before (2.28).

\section{(ii) The large volume limit}

In this limit all IIA Kähler moduli are large: $S \rightarrow \infty, T \rightarrow \infty$. This can be interpreted as having $X^{0}$ as vanishing cycle. Using the aligned flux $e_{0}$, one derives the following superpotential

$$
W=e_{0} X^{0}
$$

\section{(iii) The conifold limit}

The conifold limit is the limit where one of the IIB three-cycles $\mathcal{C}_{I I B}^{(3)}$ shrinks to zero size. As already observed in [1], this conifold limit corresponds in IIA compactification to the limit where the entire quantum six-volume of the CY vanishes, i.e.

$$
F_{0} \rightarrow 0
$$

Hence we turn on the aligned flux $m^{0}$, and the superpotential takes the form

$$
W=m^{0} F_{0}
$$

\section{(iv) The field theory Seiberg-Witten limit}

In this limit non-perturbative monopoles or dyons become massless [27]. In case of massless monopoles, $u \rightarrow 1$ corresponding to $a_{D} \rightarrow 0$, in the notation of [27]. The string interpretation of this situation is a double scaling limit, namely the intersection of the 
conifold limit with the large $S$ limit, which can be regarded as going to the $u$-plane. Hence we expect (actually more cycles vanish, see sect. 3.7)

$$
F_{0} \rightarrow 0, \quad \frac{1}{2} F_{1}+i X^{2} \rightarrow 0 .
$$

(The first line in this equation describes the conifold limit, whereas the second line corresponds to going to the $u$-plane divisor.) The corresponding superpotential with aligned fluxes will turn out to be

$$
W=m^{0} F_{0}+m\left(F_{1}+2 i X^{2}\right)
$$

Finally, vanishing dyon masses correspond to $u \rightarrow-1, a_{D}-a \rightarrow 0$. Since the sum of the dyon electric/magnetic charges plus the monopole charges equals the $W^{ \pm}$-boson charges, we can conclude that

$$
F_{0}-X^{0}+\frac{1}{-} F_{1} \rightarrow 0, \quad \frac{1}{2} F_{1}+i X^{2} \rightarrow 0 .
$$

The superpotential is

$$
W=-m^{0}\left(2 F_{0}-2 X^{0}+F_{1}\right)+m^{1}\left(F_{1}+2 i X^{2}\right) .
$$

\section{(v) The strong coupling limit}

The strong coupling singularity [28] is an example of a degeneration where two intersecting cycles shrink to zero size. In case of non-vanishing NS and Ramond fluxes this degeneration leads to a situation in which the fluxes are non-local w.r.t. each other. To be specific consider a type IIA compactification on the Calabi-Yau manifold $\mathbf{P}^{4}(1,1,2,8,12 \| 24)$ with $h^{1,1}=3$ and $h^{2,1}=243$ which is an elliptic fibration over the Hirzebruch surface $\mathbf{F}_{2}$. The three vector moduli are $S$, the volume of the $\mathbf{P}^{1}$ basis of $\mathbf{F}_{2}, U$, the volume of the $\mathbf{P}^{1}$ fiber of $\mathbf{F}_{2}$, and $T$, the volume of the elliptic fiber $E$. At the strong coupling point $S=0$ the following two cycles with non-trivial intersection number shrink to zero size:

$$
\begin{aligned}
& \operatorname{vol}\left(\mathcal{C}_{S}^{(2)}\right) \rightarrow 0 \quad \Longleftrightarrow \quad S \rightarrow 0, \\
& \operatorname{vol}\left(\mathcal{C}_{S}^{(4)}\right)-\frac{1}{2} \operatorname{vol}\left(\mathcal{C}_{U}^{(4)}\right) \rightarrow 0 \quad \Longleftrightarrow \quad F_{S}-\frac{1}{2} F_{U} \rightarrow 0,
\end{aligned}
$$

where $C_{i}^{(2)} \cap C_{j}^{(2)}=\delta_{i j}$. At this point a $U(1)$ gauge group is enhanced to $S U(2)$, and also an $S U(2)$ adjoint hypermultiplet becomes massless. Hence the corresponding $(\mathcal{N}=2)$ $\beta$-function vanishes. The superpotential with aligned fluxes is then

$$
W=i e_{S} S+i m\left(2 F_{S}-F_{U}\right)
$$




\subsection{The IIA large volume limit and the perturbative heterotic limit}

\subsubsection{The classical heterotic limit}

In this section we consider the classical heterotic limit, or equivalently in IIA language the limit where the base of the K3 fibration is large (see the appendix for more details), i.e. $S \rightarrow \infty$. In this limit the prepotential is

$$
F=i\left(X^{0}\right)^{2} S\left(T^{a} \eta_{a b} T^{b}+1\right)
$$

where $S=-i X^{1} / X^{0}, T^{a}=-i X^{a} / X^{0}$. The corresponding period vector is then $\left(X^{0}=1\right)$ $\left(X^{I}, F_{I}\right)=\left(1, i S, i T^{a} ;-i S T^{a} \eta_{a b} T^{b}+i S, T^{a} \eta_{a b} T^{b}, 2 S \eta_{a b} T^{b}\right)$.

As discussed before, we want to choose the H-fluxes aligned with the directions of the vanishing cycles $X^{0}, X^{a}$ and $F_{1}$. This leads to the following non-vanishing fluxes $e_{0}, e_{a}$, $m^{S}$, and the superpotential takes the following form:

$$
W=\tilde{e}_{0}-m^{S} T^{a} \eta_{a b} T^{b}+i e_{a} T^{a}
$$

where $\tilde{e}_{0}=e_{0}-m^{s}$. The $\mathcal{N}=2$ supersymmetric zero energy ground state, $W=0$ and $e^{\frac{K}{2}} D W=0$, is obtained for the following (attractor) values of the (real) moduli:

$$
e_{a} T^{a}=0, \quad \tilde{e}_{0}-m^{S} T^{a} \eta_{a b} T^{b}=0, \quad S=\infty .
$$

On the other hand, for finite $S$ supersymmetry is completely broken.

To be concrete, let us investigate in more detail the corresponding scalar potential for the $S T U$ model, assuming, for simplicity, that the three moduli are real:

$$
v=\frac{\tilde{e}_{0}^{2}+\left(m^{S}\right)^{2} T^{2} U^{2}+e_{T}^{2} T^{2}+e_{U}^{2} U^{2}}{2 S T U}
$$

In the directions of $T$ and $U$ this scalar potential has its minima at the $\mathcal{N}=2$ supersymmetry preserving points

$$
T_{\min }=-\frac{e_{U}}{e_{T}} U_{\min }, \quad U_{\min }= \pm \sqrt{-\frac{\tilde{e}_{0} e_{T}}{m^{S} e_{U}}},
$$

where we need $-\frac{e_{T} \tilde{e}_{0}}{m^{S} e_{U}}>0$ for real moduli fields, as we assumed here. In the direction of the $S$-field the scalar potential has no minimum, but has a run-away behavior, $v \sim 1 / S$, which drives the $S$-field to infinity.

The classical field theory limit is naturally contained in this discussion. This is the limit, where we turn off all field theory quantum effects, and the perturbative $W^{ \pm}$-bosons become massless. In string theory, this limit corresponds to a double scaling limit, 
namely to $S \rightarrow \infty$ together with $T \rightarrow U$. Specifically, within the $S T U$ model this limit is achieved by choosing $\tilde{e}_{0}=m^{S}=0$ and $e_{T}=-e_{U}$ in the superpotential eq.(3.21), i.e. $W=i e_{T}(T-U)$. The corresponding minima are at the line $T=U$, where the classical gauge group is enhanced to $S U(2)$.

In summary, using the classical heterotic prepotential and turning on aligned fluxes one finds supersymmetric minima with vanishing potential for finite $T_{a}$ and infinite $S$. In fact, for $S \rightarrow \infty$, the whole $\mathcal{N}=2$ supersymmetry is restored.

\subsubsection{The large volume limit}

In this limit all Kähler moduli $t^{A}$ are large, $\operatorname{Im}\left(t^{A}\right) \rightarrow \infty$, which means that all rational instantons are suppressed. The prepotential is determined by the intersections numbers $C_{A B C}$ and has the form

$$
F^{\mathrm{IIA}}=-\frac{1}{6} C_{A B C} \frac{X^{A} X^{B} X^{C}}{X^{0}},
$$

where the IIA Kähler moduli are defined as $t^{A}=X^{A} / X^{0}(\operatorname{Im} t>0)$. The Kähler potential is in this limit

$$
K=-\log \left(\frac{i}{6} C_{A B C}\left(t^{A}-\bar{t}^{A}\right)\left(t^{B}-\bar{t}^{B}\right)\left(t^{C}-\bar{t}^{C}\right)\right)
$$

Let us first discuss the case of aligned fluxes, i.e. $e_{0}$ is the only non-vanishing flux, which leads to the superpotential $W=e_{0} X^{0}$. This case is essentially contained in the discussion of the previous section (see eq.(3.23)). The scalar potential can be computed in a straightforward manner (see also [1]):

$$
v=4 e_{0}^{2} e^{K} \sim \frac{\left(e_{0}\right)^{2}}{\operatorname{vol}(C Y)} .
$$

This potential has no extrema for finite values of $t^{A}$, but it shows the characteristic runaway behavior, which drives all moduli to infinity, where supersymmetry is unbroken.

As an alternative let us consider a choice of Ramond fluxes which are not aligned with the vanishing cycle $\mathcal{C}^{(0)}$. Specifically, we decide to turn on the flux $e_{0}$ and all fluxes $m^{A}$, which correspond to the 4 -cycles $\mathcal{C}_{A}^{(4)}$. The superpotential is now

$$
W=X^{0}\left(e_{0}+\frac{1}{2} m^{A} C_{A B C} t^{B} t^{C}\right) .
$$

The conditions for preserving supersymmetry in the sector of the fields $t^{A}, h^{A}=0$, are then solved, using the attractor equations (2.52), by the following values of the moduli 21:

$$
t_{\text {Susy }}^{A}=-i m^{A} \sqrt{-\frac{6 e_{0}}{C_{A B C} m^{A} m^{B} m^{C}}} .
$$


(We haven chosen $e_{0}<0$ and $m^{A}>0$.) Consistency with the large volume limit requires $-e_{0} \gg m^{A}$.

For the gravitino mass $m_{3 / 2}^{2}=e^{G}$ at the points (3.29) we find

$$
\left.m_{3 / 2}^{2}\right|_{\text {Susy }}=2 \sqrt{-e_{0} \frac{C_{A B C}}{6} m^{A} m^{B} m^{C}} .
$$

Note that this expression is identical to the entropy $\frac{\mathcal{S}}{\pi}$ of classical Calabi-Yau black hole solutions which are due $m^{A}$ D4-branes, wrapped around the CY 4-cycles, plus $e_{0} \mathrm{D} 0$ branes. Since $C_{A B C} m^{A} m^{B} m^{C} \neq 0, m_{3 / 2}$ is non-zero.

Analyzing the scalar potential it turns out that the points (3.29) are indeed extrema of $v$; at these extrema the potential has the value:

$$
\left.v\right|_{\text {Susy }}=2 \sqrt{-e_{0} \frac{C_{A B C}}{6} m^{A} m^{B} m^{C}} .
$$

Although the auxiliary fields $h^{A}$ are zero at the extrema of $v$, supersymmetry is nevertheless broken in the sector of the hypermultiplets $\tau$ and $Y$. This comes from the fact that $W \neq 0$ at the points (3.29), and hence $h^{\tau}, h^{Y} \neq 0$.

To be more specific about the nature of the extrema of $v$, let us compute $v$ for the $S T U$ model with $C_{S T U}=1$ and all other $C_{A B C}=0$ for real moduli $S, T, U$ :

$$
v=\frac{e_{0}^{2}+\left(m^{1}\right)^{2} T^{2} U^{2}+\left(m^{2}\right)^{2} S^{2} U^{2}+\left(m^{3}\right)^{2} S^{2} T^{2}}{2 S T U} .
$$

We see that this potential indeed possesses a minimum at the points eq.(3.29). Therefore the model with non-aligned fluxes exhibits a stable non-supersymmetric ground state with positive scalar potential at its minimum. On the other hand, since the moduli $t^{A}$ at the minimum of the potential are large $\left(\right.$ for $-e_{0} \gg m^{A}$ ) but not infinite, one should also discuss the contribution of the exponentially suppressed instanton terms to the prepotential, where we expect that the minima of the potential are shifted by corrections of the order $e^{-t^{A}}$.

\subsection{The conifold locus}

In this section we want to explore the vacuum structure of a type IIB compactification near the conifold locus in the moduli space. The generic conifold locus is the co-dimension one locus in $\mathcal{M}$, where in $M$ a cycle, say $A_{1}$, with the topology of $S^{3}$ vanishes, while the remaining 3-cycles stay finite. More precisely the Calabi-Yau space $M$ exhibits a nodal singularity, i.e. it is described locally by the eq. $\sum_{i=1}^{4} \epsilon_{i}^{2}=\mu$. For $\mu \rightarrow 0$ the real 
part of this local equation describes the vanishing $S^{3}$. In the vicinity of a conifold point, $X^{1}=\int_{A_{1}} \Omega \rightarrow 0$, an additional hypermultiplet, the ground state of a singly wrapped 3-brane around $A_{1}$, with mass proportional to $\left|X^{1}\right|$ becomes light [29]. It is charged w.r.t. to the $U(1)^{N_{V}}$ gauge symmetry of the vector multiplets ${ }^{\mathrm{T}}$. Related $\mathcal{N}=2$ black hole solutions at the conifold locus were considered before in [29, 30].

In the following we will discuss the simplest situation with periods $X^{i}=\int_{A_{i}} \Omega, i=0,1$ and the dual periods $F_{i}=\int_{B^{i}} \Omega$ (with $A_{i} \cap B^{j}=\delta_{i}^{j}$ ), where $X^{1}$ vanishes at the conifold locus and the other periods remain finite. So at the conifold we have

$$
\mu:=\frac{X^{1}}{X^{0}}=0
$$

In addition, if the 3 -fold is transported along a closed loop around the conifold locus the period $F_{1}$ undergoes a monodromy transformation, given by the Lefshetz formula

$$
F_{1} \rightarrow F_{1}+X^{1}
$$

while all other periods have trivial monodromy. Therefore the periods near the conifold have the expansion [13 $F_{1}=\sum_{i=0}^{2} c_{1}^{(i)} \mu^{i}+\frac{X^{1}(\mu)}{2 \pi i} \log (\mu)+\mathcal{O}\left(\mu^{3}\right), X^{1}=c \mu+\mathcal{O}\left(\mu^{2}\right), F_{0}=$ $\sum_{i=0}^{2} c_{0}^{(i)} \mu^{i}+\mathcal{O}\left(\mu^{3}\right)$ and $X^{0}=\sum_{i=0}^{2} c_{(i)}^{0} \mu^{i}+\mathcal{O}\left(\mu^{3}\right)$ and near $\mu=0$ the prepotential can be expanded as

$$
F=-i\left(X^{0}\right)^{2}\left(a+\frac{c^{2}}{4 \pi} \mu^{2} \log \mu+b \mu+(\text { analytic terms })\right) .
$$

It is easy to see that the Kähler potential is finite at the conifold point:

$$
e^{-K_{V}}=4 \operatorname{Im} a
$$

In contrast, the internal moduli space metric logarithmically diverges at the conifold point:

$$
K_{\mu \bar{\mu}}=-\log |\mu| \frac{1}{2 \operatorname{Im} a}
$$

In the type IIA mirror compactification on the mirror quintic $W$ the conifold point for $M$ corresponds to the limit where the entire quantum volume of $W$ shrinks to zero size, whereas the other cycles stay finite [23]. $\chi=-200, \int c_{2} J=50$ and $n_{11}=1$ [23] fixes the integral basis (3.2). Using the relation $\mu=1-5^{5} z$, where $z$ is the variable at the

\footnotetext{
${ }^{12}$ E.g. it corresponds to a magnetic monopole or dyon in the effective gauge theory, whereas its effective supergravity description is given as a massless black hole.

${ }^{13}$ For the field theory interpretation it is essential that $c_{i}^{(0)} \neq 0$, otherwise a magnetically as well as an electrically charged particle become massless at $\mu=0$. The $\mathcal{O}\left(\mu^{n}\right)$ parts are fixed by the Picard-Fuchs equation.
} 
large complex structure point $z=0$ [24], $c$ turns out to be $\frac{\sqrt{5}}{2 \pi i}$ and one can fix the $c_{I}^{(j)}$, $j=1,2$ so that after analytic continuation" $X^{\infty, 0}=-F_{1}, X^{\infty, 1}=-F_{0}, F_{1}^{\infty}=X^{0}$ and $F_{0}^{\infty}=X^{1}$. One checks that $X^{I}, F_{I}$ satisfy the integrability conditions for the existence of the prepotential and determines the constants in $F: a=0.517061+i .04500226$ and $b=0$. Even for this choice of coordinates in one parameter models the constant $a$ is not universal, as we find for the sextic in $\mathbf{P}(1,1,1,1,2) a=.147507 i$, but $b=0$ for all one moduli cases.

For hypersurfaces in toric varieties with an arbitrary number of moduli we find that the $S^{3}$ vanishing at the principal discriminant [24] corresponds via mirror symmetry always to the quantum volume $F_{0}^{\infty}$.

Consider the case where the flux that is aligned with the vanishing cycle of the conifold point is turned on. In type IIB the corresponding superpotential is

$$
W=e_{1} \mu
$$

Since $W=0$ but $\partial W / \partial \mu=e_{1} \neq 0$ at the conifold point, one might expect that the conifold point does not correspond to a supersymmetric ground state with vanishing scalar potential. However this conclusion is not correct in the context of supergravity, since the Kähler metric diverges at the conifold point. In fact, the corresponding supergravity scalar potential in the vicinity of the conifold point,

$$
v=\left|W_{\mu}\right|^{2} e^{K} K_{\mu \bar{\mu}}^{-1}=-\frac{e_{1}^{2}}{\log |\mu|^{2}},
$$

has a supersymmetry preserving minimum at $\mu=0$ with $v=0$. Hence $\mu$ is attracted to the conifold point [1].

This ground state of supergravity is not changed if also the additional light hypermultiplet is included into the superpotential at the conifold point [3]:

$$
W=e_{1} \mu+\mu \phi \tilde{\phi}
$$

Now the supersymmetric, stationary points of $v$ are at $W=0$ and $d W=0$, which leads again to $\mu=0$ and in addition to $\phi \tilde{\phi}=-e_{1}$, as discussed in [3].

\subsection{Colliding conifold loci}

Next we study the situation where two conifold loci meet. (This corresponds to an $A_{2}$ singularity, cf. below. The generalization to $A_{n}$ singularities is straightforward.) To be

\footnotetext{
${ }^{14}$ To six significant digits, they are $c_{1}^{(0)}=1.07073, c_{1}^{(1)}=-.0247076, c_{1}^{(2)}=.0566403, c_{0}^{(0)}=6.79502-$ $7.11466 i, c_{0}^{(1)}=1.01660-.829217 i, c_{0}^{(2)}=.711623-.580451 i, c_{(0)}^{0}=1.29357 i, c_{(1)}^{0}=-.150767 i$ and $c_{(2)}^{0}=.777445 i$.
} 
concrete we consider the mirror of the $X_{18}(1,1,1,6,9)$ Calabi-Yau hypersurface, a two parameter model where two conifold loci meet [43]. Many aspects of the generalization to the meeting of several conifold loci are straightforward. $X_{18}(1,1,1,6,9)$ is an elliptic fibration over $\mathbf{P}^{2}$ and the mirror manifold may be defined by

$$
P=x_{1}^{18}+x_{2}^{18}+x_{3}^{18}+x_{4}^{3}+x_{5}^{2}-3 \Phi x_{2}^{6} x_{2}^{6} x_{3}^{6}-6^{\frac{2}{3}} \Psi x_{1} x_{2} x_{3} x_{4} x_{5}=0,
$$

with the orbifold action $Z_{18} \times Z_{18}:\left(x_{1} \mapsto x_{1} \exp \frac{2 \pi i}{18}, x_{2} \mapsto x_{2} \exp \left(17 \frac{2 \pi i}{18}\right)\right)\left(x_{1} \mapsto\right.$ $\left.x_{1} \exp \frac{2 \pi i}{18}, x_{3} \mapsto x_{3} \exp \left(17 \frac{2 \pi i}{18}\right)\right)$ on the coordinates. The canonical large complex structure coordinates are 24]

$$
\begin{array}{ll}
z_{1}=-\frac{\Phi}{\Psi^{6}}, & l_{1}=(0,0,0,2,3,1,-6), \\
z_{2}=-\frac{1}{\Phi^{3}}, & l_{2}=(1,1,1,0,0,-3,0) .
\end{array}
$$

Here the $l_{i}$ are the generators of the Mori cone, which identify the complex coordinates of the mirror near $z_{i}=0$ as $\frac{\log \left(z_{1}\right)}{2 \pi i} \sim t_{E}, \frac{\log \left(z_{2}\right)}{2 \pi i} \sim t_{\mathbf{P}^{1}}$, with the Kähler parameter $t_{E}$ of $X_{18}(1,1,1,6,9)$ measuring the size of the elliptic fiber and $t_{\mathbf{P}^{1}}$ measuring the size a $\mathbf{P}^{1}$ in the base $\mathbf{P}^{2}$. We find the discriminant by solving $\frac{\partial P}{\partial x_{i}}=P=0$ for $\Phi, \Psi$ and $x_{i}$. The principal discriminant is the solution where all $x_{i} \neq 0$. We find $x_{5}=3\left(\Psi x_{1} x_{2} x_{3}\right)^{3}$, $x_{4}=6^{\frac{1}{3}}\left(\Psi x_{1} x_{2} x_{3}\right)^{2}, x_{1}^{18}=x_{2}^{18}=x_{3}^{18} \neq 0$ and

$$
\Delta_{\text {con }_{1}}=1-\left(\Psi^{6}+\Phi\right)^{3}=0
$$

A second solution is $x_{4}=x_{5}=0, x_{1}^{18}=x_{2}^{18}=x_{3}^{18} \neq 0$ and

$$
\Delta_{\text {con }_{2}}=1-\Phi^{3}=0
$$

Near $\Psi=0$ and $\Phi=1$, where $\Delta_{c o n_{1}}=\Delta_{c o n_{2}}=0$, the local expansion of the manifold is

$$
\epsilon_{1}^{2}+\epsilon_{2}^{2}+\epsilon_{3}^{2}+\epsilon_{4}^{3}=a \epsilon_{4}+b
$$

the three-dimensional version of an $A_{2}$ singularity. In particular we have two $S_{1}^{3}$ and $S_{2}^{3}$ with $S_{1}^{3} \cap S_{2}^{3}=1$, but as three is odd we have $S_{i}^{3} \cap S_{i}^{3}=0$.

In the $z$ coordinates we have, up to irrelevant factors, $\Delta_{\text {con }}=1-3 z_{1}+3 z_{1}^{2}-\left(1+z_{2}\right) z_{1}^{3}$ and $\Delta_{\text {con }}=1+z_{2}$, so the conifolds collide at the two points $\left(z_{1}^{ \pm}=\frac{1}{2} \pm \frac{i}{6} \sqrt{3}, z_{2}=-1\right)$. At these points we can solve the Picard-Fuchs equation in the variables $x_{1}=\left(1-\frac{z_{1}}{z^{ \pm}}\right)$and $x_{2}=\frac{1+z_{2}}{1-\frac{z_{1}}{z^{ \pm}}}$. We get, as expected, two unique vanishing solutions $X^{c c, 1}=x_{1}+\mathcal{O}\left(x^{2}\right)=$ $\int_{S_{1}^{3}} \Omega$ and $X^{c c, 2}=x_{1} x_{2}+\mathcal{O}\left(\left(x_{1} x_{2}\right)^{2}\right)=\int_{S_{2}^{3}} \Omega$ with dual periods $F_{1}^{c c}=\frac{1}{2 \pi i} X^{c c, 1} \log \left(X^{c c, 1}\right)+$ $a_{1}+$ holom. $=\int_{T_{1}^{3}} \Omega$ and $F_{2}^{c c}=\frac{1}{2 \pi i} X^{c c, 2} \log \left(X^{c c, 2}\right)+a_{2}+$ holom. $=\int_{T_{2}^{3}} \Omega$. Further we 
can see, in this case by analytic continuation, that $T_{1}^{3} \cap T_{2}^{3}=0$ and furthermore that the remaining two periods $X^{c c, 0}, F_{0}^{c c}$ start with a constant term. This implies that to leading order the Weil-Petersson-metric near the conifold is

$$
G_{x_{i}, \bar{x}_{j}}=-\left(\begin{array}{cc}
c_{1} \log \left(\left|x_{1}\right|\right) & 0 \\
0 & c_{2} \log \left(\left|x_{2}\right|\right)
\end{array}\right),
$$

with $c_{1}, c_{2}>0$. The relation between $X_{i}^{c c}$ and the periods at infinity can be obtained via analytic continuation. One finds $X^{c c, 1} \propto F_{0}^{\infty}$ and $X^{c c, 2} \propto F_{E}^{\infty}-3 F_{\mathbf{P}^{1}}^{\infty}-X^{\infty, 0}$. It follows that we can turn on fluxes which lead to a superpotential $W=n_{1} X^{c c, 1}+n_{2} X^{c c, 2}$ and a scalar potential which is in leading order

$$
v=-a_{1} \frac{n_{1}^{2}}{\log \left(\left|x_{1}\right|\right)}-a_{2} \frac{n_{2}^{2}}{\log \left(\left|x_{2}\right|\right)},
$$

where $a_{1}, a_{2}>0$. Note that we have chosen the flux vector such that it fixes the minimum of the potential in the moduli space at complex codimension two.

\subsection{The Seiberg-Witten limit}

In the type II geometry, the Seiberg-Witten $S U(2)$ theory emerges at the blow up in the moduli space, which resolves the tangency of the weak coupling divisor $y \propto e^{-S}=0$ and the generic conifold locus. The simplest situation where this geometry arises is for the 2 moduli $K_{3}$ fibration examples studied in [25, 24, 16]. A schematic picture of the moduli space for this type of models can be found in Fig. 4 of [25]. As shown in Fig. 1 below we will use a slightly different resolution of the tangency of $\Delta_{\text {con }}$ and $y=0$ than refs. [25, 16]. The advantage is that this resolution splits the monopole and the dyon point, which is important when writing down the superpotential. Furthermore the SW-monodromy group is embedded in the simplest possible way in the Calabi-Yau monodromy group. In [4] model independent general expressions for the scalar potential have been obtained at the Seiberg-Witten point. Here we will derive the scalar potential for a specific model.

In particular, for the well studied degree 12 hypersurface in $\mathbf{P}(1,1,2,2,6)$ we have $C_{T T T}=$ 4, $C_{S T T}=2$, zero otherwise, and $\int_{W} c_{2} J_{T}=52, \int_{W} c_{2} J_{S}=24$ and $\chi=-252$. Here we denote the Kähler class measuring the complex volume of the $\mathbf{P}^{1}$ basis of the $K_{3}$ fibration by $S$ and the square root of the complex volume of the $K_{3}$ by $T$. The integral basis is fixed by (3.2). The connection to the canonical large complex structure variables is given by the leading order relations $z_{t} \propto e^{-T}, z_{s} \propto e^{-S}$. We use the same rescaled complex structure variables as in [24], $x=1728 z_{t}$ and $y=4 z_{s}$. The order two tangency between the conifold divisor $\Delta_{c o n}=\left\{\Delta_{+}^{c o n} \Delta_{-}^{c o n}=((1-x)+x \sqrt{y})((1-x)-x \sqrt{y})=(1-x)^{2}-y x^{2}=0\right\}$ 
and the weak coupling singularity $W=\{y=0\}$ is now resolved by blowing up which introduces an exceptional divisor $E \sim \mathbf{P}^{1}$, see the right-hand side of Fig. 1. Near $W \cap E$ the dimensionless variables $w_{1}=\frac{x \sqrt{y}}{(1-x)}=\frac{1}{\tilde{u}}$ and $w_{2}=(1-x)=\alpha^{\prime} u+\mathcal{O}\left(\alpha^{\prime 2}\right)$ are good coordinates. Here $\tilde{u}=\frac{u}{\Lambda^{2}}$ is dimensionless of order one and the identification with the Seiberg-Witten variables to first order in $\alpha^{\prime}$ is dictated by the double scaling limit [16] $\epsilon \rightarrow 0$ in

$$
y=e^{-S}=:\left(\alpha^{\prime}\right)^{2} \Lambda^{4} e^{-\hat{S}}=: \epsilon^{4}, \quad(1-x)=\alpha^{\prime} u+\mathcal{O}\left(\alpha^{\prime 2}\right)=\epsilon^{2} \tilde{u}+\mathcal{O}\left(\epsilon^{4}\right)
$$

The last equation implies in particular $\sqrt{\alpha^{\prime}} \Lambda=\frac{\Lambda}{M_{S t r}}=\epsilon$. Since $\tilde{u} e^{-S / 2} \sim(1-x)$ and $(1-x)$, is proportional at weak coupling to the mass square of $W^{ \pm}$in string units, the exponential relation in the double scaling limit is a reflection of the renormalization group equation $\frac{8 \pi^{2}}{b_{1} g^{2}\left(M_{S t r}\right.}=-\log \left(\frac{m_{W^{ \pm}}}{M_{S t r}}\right)$ due to the exact one-loop $\beta$-function of $\mathcal{N}=2$ with coefficient $b_{1}=4$ for pure $S U(2)$ Yang-Mills theory.

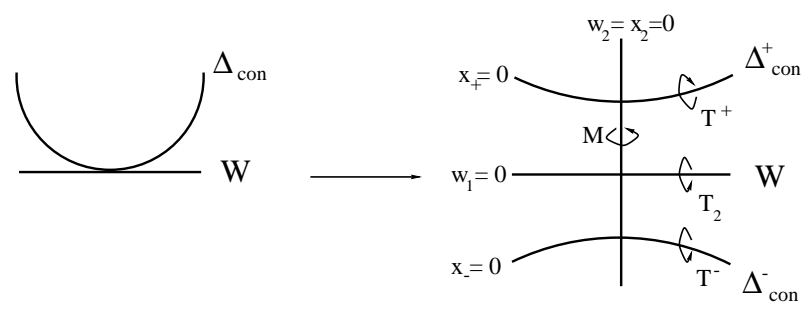

E

Fig. 1 Blow up of the Seiberg-Witten point.

The Picard-Fuchs equation in [24] can be easily solved in the $\left(w_{1}, w_{2}\right)$ coordinates near $W \cap E$. This corresponds to the classical field theory limit in sect. 3.4 .1 (considered there for the $S T U$ model.

$$
\begin{aligned}
\Xi_{u=\infty}^{1}=1+\mathcal{O}\left(\left(\alpha^{\prime} u\right)^{2}\right) & =1-\frac{5}{216}\left(1+\frac{1}{2} w_{1}^{2}\right) w_{2}^{2}+\mathcal{O}\left(w_{2}^{3}\right) \\
\Xi_{u=\infty}^{2}=\alpha^{\prime} u+\mathcal{O}\left(\left(\alpha^{\prime} u\right)^{2}\right) & =w_{2}-\frac{77}{108}\left(1+\frac{1}{2} w_{1}^{2}\right) w_{2}^{2}+\mathcal{O}\left(w_{2}^{3}\right) \\
\Xi_{u=\infty}^{3}=\sqrt{\alpha^{\prime}} a(\tilde{u})\left(1+\mathcal{O}\left(\alpha^{\prime} u\right)\right) & =\frac{i}{\pi} \sqrt{w_{2}}\left(1-\frac{1}{16} w_{1}^{2}-\frac{15}{1024} w_{1}^{4}+\ldots\right)+\mathcal{O}\left(w_{2}^{3 / 2}\right) \\
\Xi_{u=\infty}^{4}=s\left(1+\mathcal{O}\left(\left(\alpha^{\prime} u\right)^{2}\right)\right. & =\frac{\Xi_{u=\infty}^{1}}{\pi i} \log \left(w_{1} w_{2}\right)-\frac{32}{81} w_{2}^{2}+\mathcal{O}\left(w^{3}\right) \\
\Xi_{u=\infty}^{5}=\alpha^{\prime} u s\left(1+\mathcal{O}\left(\alpha^{\prime} u\right)\right) & =\frac{\Xi_{u=\infty}^{2}}{\pi i} \log \left(w_{1} w_{2}\right)-\frac{139}{81} w_{2}^{2}+\mathcal{O}\left(w^{3}\right) \\
\Xi_{u=\infty}^{6}=\sqrt{\alpha^{\prime}} a_{D}(\tilde{u})\left(1+\mathcal{O}\left(\alpha^{\prime} u\right)\right) & =\frac{\Xi_{u=\infty}^{3}}{\pi i} \log \left(w_{1}\right)+\frac{2 \sqrt{w_{2}}}{\pi^{2}}\left(1-\frac{1}{8} w_{1}^{2}+\mathcal{O}\left(w_{1}^{4}\right)\right)+\kappa \Xi_{u=\infty}^{3}
\end{aligned}
$$


with $\kappa=\frac{i}{\pi}(3 \log (2)-1)$. Classical gauge group enhancement to $S U(2)$ occurs at $a=0$ where the period $\Xi_{u=\infty}^{3}$ vanishes.

Near the conifold branch $\Delta_{+}^{\text {con }} \cap E, x_{+}=\frac{1-x}{x \sqrt{y}}-1=\tilde{u}-1$ and $x_{2}=(1-x)=\alpha^{\prime} u+\mathcal{O}\left(\alpha^{\prime 2}\right)$ are good coordinates. Near the branch $\Delta_{-}^{\text {con }} \cap E$ we may chose $x_{-}=\frac{1-x}{x \sqrt{y}}+1=\tilde{u}+1$ and $x_{2}=(1-x)=\alpha^{\prime} u+\mathcal{O}\left(\alpha^{\prime 2}\right)$. The leading terms of the above basis in the $\left(x_{+}, x_{2}\right)$ coordinates near $\Delta_{+}^{\text {con }} \cap E$ read四

$$
\begin{aligned}
\Xi_{\text {mon }}^{1}=1+\mathcal{O}\left(\left(\alpha^{\prime} u\right)^{2}\right) & =1-\frac{5}{144}\left(1+\frac{2}{3} x_{+}\right) x_{2}^{2}+\mathcal{O}\left(x_{2}^{3}\right) \\
\Xi_{\text {mon }}^{2}=\alpha^{\prime} u+\mathcal{O}\left(\left(\alpha^{\prime} u\right)^{2}\right) & =x_{2}-\frac{77}{72}\left(1+\frac{2}{3} x_{+}\right) x_{2}^{2}+\mathcal{O}\left(x_{2}^{3}\right) \\
\Xi_{\text {mon }}^{3}=\sqrt{\alpha^{\prime}} a(\tilde{u})\left(1+\mathcal{O}\left(\alpha^{\prime} u\right)\right) & =\frac{i \Xi_{m o n}^{6}}{2 \pi} \log \left(x_{+}\right)+\frac{\sqrt{2 x_{2}}}{i \pi^{2}}\left(1-\frac{46}{81} x_{2}+\mathcal{O}\left(x^{2}\right)\right)+\delta \Xi_{m o n}^{6} \\
\Xi_{\text {mon }}^{4}=s\left(1+\mathcal{O}\left(\left(\alpha^{\prime} u\right)^{2}\right)\right. & =\frac{\Xi_{m o n}^{1}}{\pi i} \log \left(x_{2}\right)-\frac{1}{\pi i} \log \left(1-x_{+}\right)+\mathcal{O}\left(x_{2}^{2}\right) \\
\Xi_{\text {mon }}^{5}=\alpha^{\prime} u s\left(1+\mathcal{O}\left(\alpha^{\prime} u\right)\right) & =\frac{\Xi_{m o n}^{2}}{\pi i} \log \left(x_{2}\right)-\frac{x_{2}}{\pi i} \log \left(1-x_{+}\right)+\mathcal{O}\left(x_{2}^{2}\right) \\
\Xi_{\text {mon }}^{6}=\sqrt{\alpha^{\prime}} a_{D}(\tilde{u})\left(1+\mathcal{O}\left(\alpha^{\prime} u\right)\right) & =\frac{1}{\sqrt{2} \pi} \sqrt{x_{2}}\left(x_{+}+\frac{9}{32} x_{+}^{2}+\frac{75}{256} x_{+}^{3}+\ldots\right)+\mathcal{O}\left(x_{2}^{\frac{3}{2}}\right),
\end{aligned}
$$

with $\delta=1+\frac{3 i(1-2 \log (2))}{\pi}$. Note that $s=2 \pi i S \propto \frac{2 \pi i}{g^{2}}$ and $-S=\log (y)$.

In this simple model we can give, at least numerically, a complete account of how the periods in the Seiberg-Witten field theory limit are related to the ones in the large complex structure basis, in which our flux choices are made, by calculating the transformation matrix $\Xi_{u=\infty}=\Xi_{m o n}=N \Xi_{\infty}$, where the basis at infinity is as in (3.2), $\Xi_{\infty} \propto\left(1, t, s, \partial_{s} F, \partial_{t} F, 2 F-s \partial_{s} F-t \partial_{t} F\right)$

$$
N=\left(\begin{array}{cccccc}
0 & i A_{+} B & 0 & \frac{A_{-} B}{2} & 0 & 0 \\
0 & i B & 0 & \frac{B}{2} & 0 & 0 \\
1 & 0 & 0 & -\frac{1}{2} & 0 & 0 \\
0 & v_{2}+i A_{-} B & A_{-} B & \frac{A_{-} B}{2}+i v_{1} & -\frac{i A_{+} B}{2} & -\frac{A_{-} B}{2} \\
0 & v_{3}+i B & B & \frac{B}{2}+i v_{4} & \frac{i B}{2} & \frac{B}{2} \\
0 & 0 & 0 & 0 & 0 & 1
\end{array}\right)
$$

with $A_{ \pm}=\frac{1}{36 \pi^{4}}\left(5 \pi^{4} \pm 12 \Gamma^{8}\left(\frac{3}{4}\right)\right), B=-\frac{\pi^{3} \sqrt{3}}{\Gamma^{4}\left(\frac{3}{4}\right)}, v_{1} \approx-4.0767326, v_{2} \approx-16.409393$, $v_{3} \approx-69.6002844$ and $v_{4} \approx-8.61884321$. From the third and last line in $N$ one sees

${ }^{15}$ Note that $w_{1}^{1 / 2} w_{2}^{1 / 2}=\left(x_{+}+1\right)^{1 / 2} w_{2}^{1 / 2}=\sqrt{\alpha^{\prime}} \Lambda$, has to be factored out from the solutions on the right to obtain the Seiberg-Witten expansions.

${ }^{16}$ Explicit results for this Calabi-Yau manifold had already been obtained by W. Lerche and P. Mayr (unpublished notes). 
that the periods, which contain the Seiberg-Witten periods in the normalization $\square(a \sim$ $\left.\frac{1}{2} \Lambda_{s w} \sqrt{2 \tilde{u}}, a_{D} \sim \frac{2 i}{\pi} a \log (\tilde{u})\right)$, have intersection 1 so we can make them dual in a symplectic basis, but because of the entry $-\frac{1}{2}$ not in an integral symplectic basis.

The monodromy in the above basis around $x_{+}=0$ can be identified directly with the Seiberg-Witten monopole monodromy $\tilde{M}_{(0,1)}$ while the combination of monodromies around $w_{2}=0$ and $w_{1}=0$ give the the Seiberg-Witten monodromy around infinity $\tilde{M}_{\infty}=M T_{2}^{-1}$

$$
\tilde{M}_{\infty}=\left(\begin{array}{rrrrrr}
1 & 0 & 0 & 0 & 0 & 0 \\
0 & 1 & 0 & 0 & 0 & 0 \\
0 & 0 & -1 & 0 & 0 & 0 \\
0 & 0 & 0 & 1 & 0 & 0 \\
0 & 0 & 0 & 0 & 1 & 0 \\
0 & 0 & 4 & 0 & 0 & -1
\end{array}\right) \quad \tilde{M}_{(0,1)}=\left(\begin{array}{rrrrrr}
1 & 0 & 0 & 0 & 0 & 0 \\
0 & 1 & 0 & 0 & 0 & 0 \\
0 & 0 & 1 & 0 & 0 & -1 \\
0 & 0 & 0 & 1 & 0 & 0 \\
0 & 0 & 0 & 0 & 1 & 0 \\
0 & 0 & 0 & 0 & 0 & 1
\end{array}\right) .
$$

It is a check on $N$ that these are integral in the basis (3.2) and can be identified with $\tilde{M}_{(0,1)}=T$ and $\tilde{M}_{\infty}=A^{-1} T A T$ in the notation of [16]. Similarly, the monodromy around $x_{-}$gives $\tilde{M}_{(-1,1)}=\tilde{M}_{(0,1)}^{-1} \tilde{M}_{\infty}$.

Let us first discuss the superpotential which arises in the classical field theory limit,

$$
W=n \Xi_{u=\infty} \sim n \sqrt{\alpha^{\prime}} a
$$

which vanishes at the point of classical gauge group enhancement. Using the third row of the matrix $N$, we see that

$$
\Xi_{u=\infty}^{3}=X_{\infty}^{0}-\frac{i}{2} \frac{\partial F}{\partial S}
$$

and hence the superpotential becomes

$$
W=n\left(1-\frac{i}{2} \frac{\partial F}{\partial S}\right)=\frac{n}{2}\left(1-T^{2}\right) .
$$

This superpotential matches exactly with the superpotential in (3.10) after setting $e_{0}=$ $-2 m^{1}=n$ and $e_{2}=0$.

Let us now go to the point where the monopole becomes massless. We first discuss the field theory expectations and assume as in [3] that there is flux such that the field theory superpotential behaves in leading order at the Seiberg-Witten point as $W \sim m u$ [27]. This corresponds to a mass term for the adjoint scalar, which breaks $\mathcal{N}=2$ to $\mathcal{N}=1$. Roughly speaking, such a potential should be generated by a flux that has $m$

\footnotetext{
${ }^{17}$ Note that $\Lambda$ is rescaled by a numerical factor of order one, $\Lambda=\frac{\pi}{i \sqrt{2}} \Lambda_{s w}$.
} 
"units" on $\Xi_{m o n}^{2}$, i.e. $W=m X_{m o n}^{2}$. The superpotential has dimension three and since the parameters $x_{+}, x_{2}$ are dimensionless it reads in natural units as

$$
W=\frac{m}{\left(\alpha^{\prime}\right)^{\frac{3}{2}}} X_{\text {mon }}^{2} \sim \frac{m}{\left(\alpha^{\prime}\right)^{\frac{3}{2}}} x_{2}+\mathcal{O}\left(x^{2}\right)
$$

Under the double scaling limit it behaves hence as $W \sim m M_{s t r} \Lambda^{2} \tilde{u}$.

In the $\mathcal{N}=1$ field theory one expects $h$ vacua with a mass gap, where $h$ is the dual Coxeter number of the gauge group. At each vacuum there is a superpotential

$$
W_{k}=w^{k} e^{-S / h}
$$

where $w^{h}=1, k=0, \ldots, h-1$ and $S=\frac{1}{g^{2}}$. Indeed we find that $\Xi_{m o n}^{2} \sim x_{2}$ and from the period $\Xi_{\text {mon }}^{4}$ we learn that $x_{2} \sim e^{-\frac{S}{2}}$, so that the string embedding delivers precisely the right behavior of the superpotential.

The main issue will be the degeneration of the factor $e^{K} G^{x_{i} \bar{x}_{j}}$ and whether the the scalar potential drives the theory towards the Seiberg-Witten point. With the inverse of $N$

$$
N^{-1}=\left(\begin{array}{cccccc}
C & -C_{+} & \frac{1}{2} & 0 & 0 & 0 \\
i C & -i C_{-} & 0 & 0 & 0 & 0 \\
C+i u_{3} & C_{+}+i u_{4} & 0 & C & -C_{+} & \frac{1}{2} \\
2 C & -2 C_{+} & 0 & 0 & 0 & 0 \\
u_{1}+2 i C & u_{2}-2 i C_{-} & 0 & -2 i C & 2 i C_{-} & 0 \\
0 & 0 & 0 & 0 & 0 & 1
\end{array}\right)
$$

where $C=\frac{\sqrt{3} \pi}{2 \Gamma\left(\frac{3}{4}\right)^{4}}$ and $C_{ \pm}=\frac{\sqrt{3}}{72 \Gamma\left(\frac{3}{4}\right)^{4}}\left(5 \pi^{4} \pm 12 \Gamma\left(\frac{3}{4}\right)^{8}\right), u_{1} \approx 5.5157560, u_{2} \approx-.05616975$, $u_{3} \approx .1051578$ and $u_{4} \approx .263801$, we find that the Kähler factor $e^{K}$ diverges at $E \cap \Delta_{+}^{\text {con }}$ as

$$
e^{K}=\frac{1}{2} \frac{\pi}{\pi\left(2 u_{3}+u_{1}\right)-4 C \log \left(\left|x_{2}\right|\right)}-\frac{\operatorname{Re}\left(x_{2}\right) a-4 C^{2} \pi \operatorname{Re}\left(x_{+}\right)}{\left(\pi\left(2 u_{3}+u_{1}\right)-4 C \log \left(\left|x_{2}\right|\right)\right)^{2}}+\mathcal{O}\left(x^{2}\right),
$$

with $a=\pi\left(2 u_{3} C_{-}-C\left(2 u_{4}+u_{2}\right)+C_{+} u_{1}\right)$. To leading order the inverse metric is

$$
G^{x_{i} \bar{x}_{j}}=4 \pi^{2} C \frac{\pi\left(2 u_{3}+C\right)-4 C \log \left(\left|x_{2}\right|\right)}{6 \log (2)-\log \left(\left|x_{+}\right|\right)}\left(\begin{array}{cc}
\frac{1}{\left|x_{2}\right|} & -\sqrt{\frac{x_{2}}{\bar{x}_{2}}} \\
-\sqrt{\frac{\bar{x}_{2}}{x_{2}}} & \left|x_{2}\right|
\end{array}\right) .
$$

The scalar potential, to leading order in $\left(x_{+}, x_{2}\right)$, is

$$
\begin{gathered}
v=m^{2} \frac{2 \pi^{3}}{\left(\alpha^{\prime}\right)^{2}}\left(\left|x_{2}\right|^{2}\left[\pi^{2}\left(u_{1}+2 u_{3}\right)^{2}+16 C^{2} \log \left(\left|x_{2}\right|\right)^{2}-8 \pi C\left(2 u_{3}+1\right) \log \left(\left|x_{2}\right|\right)\right]-\right. \\
\left.4\left(\operatorname{Im}\left(x_{2}\right)\right)^{2}\left[2 C\left(2 C+\pi\left(u_{1}+2 u_{3}\right)\right)-8 C^{2} \log \left(\left|x_{2}\right|\right)\right]\right) \times \\
\left(\left|x_{2}\right|\left(6 \log (2)-\log \left(\left|x_{+}\right|\right)\right)\left(\pi\left(u_{1}+2 u_{3}\right)-4 C \log \left(\left|x_{2}\right|\right)\right)^{2}\right)^{-1}
\end{gathered}
$$


We have plotted $v$ in Fig. 2 .

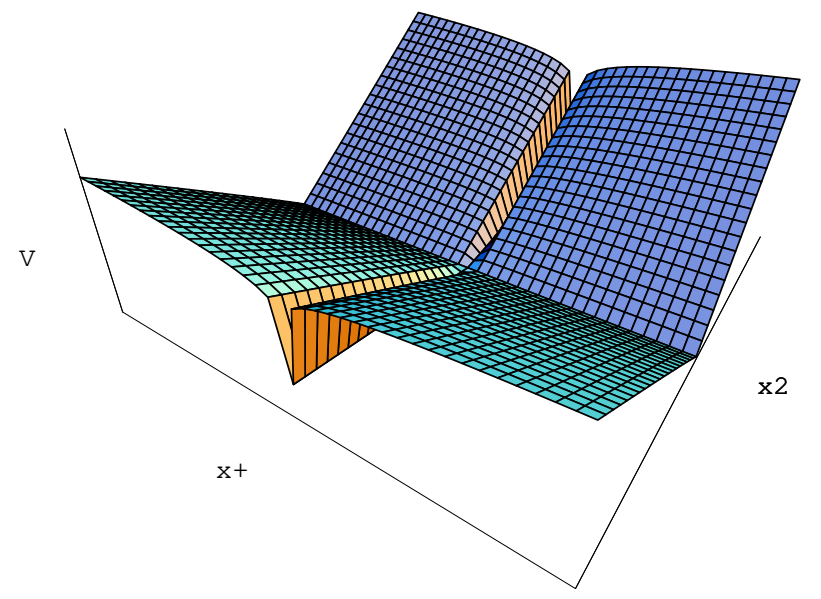

Fig. 2 Potential near the Seiberg-Witten point $x_{+}=x_{2}=0$ for the flux along $\Xi_{m o n}^{2}$.

The potential exhibits to this order two flat directions at $x_{+}=0$ and at $x_{2}=0$ along which $v=0$. Similar to the conifold case we find that there is a supersymmetric vacuum despite the fact that $\frac{\mathrm{d} W}{\mathrm{~d} x_{2}} \neq 0$ at $x_{2}=0$ due to the degeneration of the metric and the $e^{K}$ factor. Due to the $1 / \log \left(\left|x_{+}\right|\right)$term the first derivative in the $x_{+}$direction becomes infinite at $x_{+}=0$. So the potential drives the theory strongly to $x_{+}=0$. At $x_{+}=0$ the flatness in the $x_{2}$ is lifted at order $\left|x_{2}\right|^{2}$. We find at this order in $\left|x_{2}\right|^{2}$ a term

$$
\lim _{x_{+}=0, x_{2} \rightarrow 0} v=-m^{2} \frac{\pi\left|x_{2}\right|^{2}}{2\left(\alpha^{\prime}\right)^{2} C^{2}} \log \left(\left|x_{2}\right|\right)=-m^{2} \Lambda^{4} \frac{\pi|\tilde{u}|^{2}}{2 C^{2}}(\log (|\tilde{u}|)+\text { const. }) \text {. }
$$

It is worth noting that in the double scaling limit this first nonzero contribution of the expansion of the potential has the expected scale $\Lambda^{4}$ of the field theory potential.

Now let us discuss in more detail the transition from the period $\Xi_{\text {mon }}^{2}$ to the periods $\Xi_{\infty}$ at infinity, which is necessary since the integral fluxes are defined only with respect to $\Xi_{\infty}$. The fact, which allowed for the precise identification of the above flux, is that there is a unique period $\Xi_{\text {mon }}^{2}$ that behaves like $x_{2}+\mathcal{O}\left(x_{2}^{2}\right)=\alpha^{\prime} u+\mathcal{O}\left(\left(\alpha^{\prime} u\right)^{2}\right)$ in the double scaling limit. The analytic continuation (3.58) of this period to infinity (3.2) reads

$$
\Xi_{m o n}^{2}=B T+\frac{i}{2} B \frac{\partial F}{\partial S}
$$

The flux which leads to $W \sim m u$ term hence corresponds to a electric charge $e_{2}$ and a magnetic charge $m^{1}$. The irrational number $B$ can be absorbed in the definition of $m$, namely the integer fluxes are $m^{1}=\frac{1}{2} m B$ and $e_{2}=-i m B$. Note that the relative factor

\footnotetext{
${ }^{18}$ There are further terms subleading in $\log \left(\left|x_{2}\right|\right), \log \left(\left|x_{+}\right|\right)$. E.g. the $e^{K} W \bar{W}$ term contributes at this order with $\frac{1}{2}\left(\frac{\pi}{\pi\left(2 u_{3}+u_{1}\right)-4 C \log \left(\left|x_{2}\right|\right)}\right)\left|x_{2}\right|^{2}$.
} 
between the flux vector entries is $i / 2$. This means that the superpotential $W=m u$ cannot be generated by a Ramond flux alone. Specifically, whereas $m^{1}$ is a real Ramond flux, the electric flux $e_{2}$, which corresponds to the field $T$, is purely imaginary and hence is generated by a NS flux, where we have chosen the complex field $\tau$ to be imaginary, $\tau=i$.

This choice of fluxes can be compared to previous discussions in the literature on this issue. First, the identification of the flux direction as $\frac{\partial F}{\partial S} \sim u$ in [3] ignores the mixing by the analytic continuation. Second, the above identification has no zero-brane charge as in [4]. This is explained by the fact that the basis used here differs by a integral symplectic transformation relative to the one used in [4], i.e. our charge definitions are different.

If we just turn on the flux $m^{0}$ the leading behaviour of the scalar potential is

$$
v=-\frac{\pi}{2\left(\alpha^{\prime}\right)^{2}\left(\log \left(\left|x_{+}\right|\right)+6 \log (2)\right)} .
$$

This drives the theory to the conifold line, where the potential vanishes .

We have also computed the leading $x_{2}$ correction; its expression is very complicated. It is interesting that in higher order the $x_{2}$ direction is lifted so that the theory is driven towards the Seiberg-Witten point. We have plotted the potential to $\mathcal{O}\left(x^{2}\right)$ in fig. 3 .

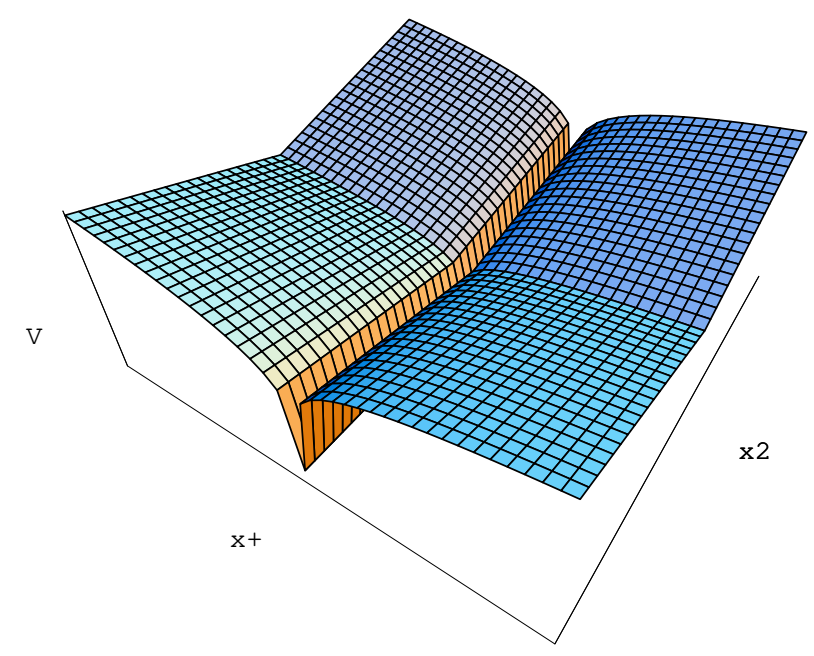

Fig. 3 Potential to order $\mathcal{O}\left(x^{2}\right)$ near the Seiberg-Witten point $x_{+}=x_{2}=0$ with flux on $F_{0}$. As already explained, besides going to the $u$-plane, the Seiberg-Witten limit also requires going to the conifold limit, $F_{0} \rightarrow 0$, where a non-perturbative monopole hypermultiplet becomes massless. Therefore we can also turn on the corresponding flux $m^{0}$. (We could also turn on fluxes along $X_{m o n}^{3}, X_{m o n}^{5}$, which also vanish.) Performing these two limits and including the mass term for the light monopole hypermultiplet $\phi_{M}$ and $\tilde{\phi}_{M}$, the 
superpotential becomes

$$
W=-m^{0} F_{0}+m\left(i \frac{\partial F}{\partial S}+2 T\right)+F_{0} \phi_{M} \tilde{\phi}_{M}
$$

Minimization of the corresponding potential will relate the vev of the monopole hypermultiplet $\phi_{M}$ to the vector moduli fields $u$ and $S$.

Let us briefly comment on the possibility of partial supersymmetry breaking from $\mathcal{N}=2$ to $\mathcal{N}=1$ at the Seiberg-Witten point. As explained in [3] partial supersymmetry breaking is possible in the rigid field theory limit after freezing out the $S$-field. From the local supergravity perspective partial supersymmetry breaking seems possible since the flux vector $e_{2}$ is imaginary, as soon as we freeze out the hypermultiplet $\tau$. Then the difference in the mass eigenvalue of the two gravitini is proportional to $2 m B T$. But if we treat $\tau$ as a dynamical field, the minimization of the potential with respect to $\tau$ will make partial supersymmetry breaking impossible, as explained before.

\subsection{The strong coupling limit and the rôle of the $\beta$-function.}

The heterotic dilaton $s$ corresponds in the dual $K 3$ fibered Calabi-Yau spaces to the complexified volume of the $\mathbf{P}^{1}$ base. Its dependence on its mirror dual complex structure modulus is governed by an universal differential equation $\left(\theta=y \frac{\mathrm{d}}{\mathrm{d} y}, y=4 z_{s}\right.$ ) (see e.g. 24

$$
\left[\theta^{2}-\frac{y}{4} \theta\left(\theta+\frac{1}{2}\right)\right] \Xi=0
$$

with three regular singular points at $y=(0,1, \infty)$. Eq. (3.66) implies, that if the other moduli of the Calabi-Yau $z_{i}$ are set to 0 , the complexified volume $s$ is given by

$$
s=\frac{1}{\pi} \arctan (\sqrt{y-1}) .
$$

In particular at $y=1$ the period ratio $s$ vanishes with $w_{s}=1-y$ as $s=\frac{1}{\pi} \sqrt{w_{s}} \sum_{n=0}^{\infty} \frac{w_{s}^{n}}{2 n+1}$. In the heterotic string this corresponds to the strong coupling limit. We therefore refer to the $w_{s}=1-y=0$ locus in the moduli space as strong coupling divisor. Inside the Calabi-Yau one finds a divisor $F$ which is a rational fibration with the $\mathbf{P}^{1}$ as fiber and a genus $g$ curve $C_{g}$ as base. If the fiber $\mathbf{P}^{1}$ vanishes, $F$ collapses to $C_{g}$ and branes wrapped on $\mathbf{P}^{1}$ yield in type IIA compactifications charged massless vector multiplets, which complete the $U(1)$ vector multiplet $s$ to a $S U(2)$ vector multiplet. The square root branch cut $\sqrt{w_{s}}$ will generate the Weyl reflection as monodromy. Holomorphic one-forms of $C_{g}$ lead to additional matter multiplets, likewise in the adjoint representation [28]. We will investigate superpotentials at the strong coupling singularity. An example is the $X_{12}(1,1,2,2,6)$ model discussed in the last section. The curve $C_{g}$ is the vanishing 
locus of the first two variables and is hence represented by $X_{6}(1,1,3)$. By the adjunction formula $g=(2-\chi) / 2=1-\left.\frac{(1+J)^{2}(1+3 J) 6 J}{2 \cdot 3(1+6 J)}\right|_{J^{2}}=2$. With two hypermultiplets in the adjoint representation the coupling grows with the scale.

At the point $\left(x=1728 z_{t}, y\right)=(0,1)$ we have in the $\left(z_{t}, w_{s}=1-y\right)$ coordinates the solutions

$\Xi_{s}^{1}=1+\frac{5}{72} z_{t}+\mathcal{O}(2)$

$\Xi_{s}^{2}=\frac{1}{2 \pi i}\left(\Xi_{s}^{1} \log \left(z_{t}\right)+\frac{1}{2} z_{t}+\frac{1}{2} \log \left(1-w_{s}\right)+\mathcal{O}(2)\right)=: \frac{1}{2 \pi i}\left(\Xi_{s}^{1} \log \left(z_{t}\right)+\Sigma_{1}\right)$

$\Xi_{s}^{3}=\frac{i}{\pi} \sqrt{w_{s}}\left(\frac{\arctan \left(\sqrt{-w_{s}}\right)}{i \sqrt{w_{s}}}+\frac{5}{216} z_{t} w_{s}+\mathcal{O}(2)\right)=a\left(w_{s}\right)+\mathcal{O}\left(z_{t}\right)$

$\Xi_{s}^{4}=\frac{\Xi_{s}^{3}}{2 \pi i} \log \left(z_{t} w_{s}\right)-\frac{\sqrt{w_{s}}}{2 \pi^{2}}\left(\frac{5}{18} w_{s}+\mathcal{O}(2)\right)=a_{D}+\mathcal{O}\left(z_{t}\right)$

$\Xi_{s}^{5}=\frac{1}{2 \pi^{2}}\left(\Xi_{s}^{1} \log \left(z_{t}\right)^{2}+2 \Sigma_{1} \log \left(z_{t}\right)-w_{s}+\frac{13}{18} x+\mathcal{O}(2)\right)=: \frac{1}{2 \pi^{2}}\left(\Xi_{s}^{1} \log \left(z_{t}\right)^{2}+2 \Sigma_{1} \log \left(z_{t}\right)+\Sigma_{2}\right)$

$\Xi_{s}^{6}=\frac{2}{3(2 \pi i)^{3}}\left(\Xi_{s}^{1} \log \left(z_{t}\right)^{3}+3 \Sigma_{1} \log \left(z_{t}\right)^{2}+3 \Sigma_{2} \log \left(z_{t}\right)-6 w_{s}+\mathcal{O}(2)\right)$.

This basis is related to the basis at $z_{t}=z_{s}=0$ by the matrix $\Pi_{\infty}=N_{s t r} \Pi_{s}$ with (cf. footnote 16)

$$
N_{s t r}=\left(\begin{array}{cccccc}
1 & 0 & 0 & 0 & 0 & 0 \\
i b_{0} & 1 & -\frac{1}{2} & 0 & 0 & 0 \\
0 & 0 & 1 & 0 & 0 & 0 \\
2 b_{1} & -4 i b_{0} & 0 & 1 & 0 & 0 \\
b_{1} & -2 i b_{0} & i b_{3} & \frac{1}{2} & 1 & 0 \\
-i b_{2} & b_{4} & 0 & -i b_{0} & 0 & 1
\end{array}\right),
$$

Here $b_{0}=\frac{\log (2)}{2 \pi}$ and $b_{1} \approx 1.09550, b_{2} \approx 1.00245, b_{3} \approx .20799$ and $b_{4} \approx 2.14233$.

We consider a flux on the vanishing period $\Xi_{s}^{3}=s$, which generates a superpotential

$$
W=n s
$$

and find the potential in leading order in $\left(z_{t}, w_{s}\right)$

$$
v=\frac{n^{2} \pi}{\left(\alpha^{\prime}\right)^{2}\left(2 \pi b_{3}-2 \log \left(\left|w_{s}\right|\left|z_{t}\right|\right)-2\right)}+\mathcal{O}(1) .
$$

This potential exhibits a similar logarithmic behaviour as the one at the conifold or the pure $S U(2)$ point. Its value is zero at $w_{s}=0$ and at $x=0$. This value represents a local 
minimum and the theory is strongly (by an infinite slope) attracted to $\left\{w_{s}=0=x=0\right\}$. The $\log \left(\left|w_{s}\right|\left|z_{t}\right|\right)$ dependence is due to the non-vanishing of the $\beta$-function of the gauge coupling constant. We thus expect a qualitative new behaviour of the scalar potential at conformal points where the $\beta$-function vanishes.

\subsection{The conformal points}

We can study the situation with vanishing $\beta$-function in the $X_{24}(1,1,2,8,12) K 3$ fibration. Here $C_{g}$ is obtained, as before, by setting to zero the first two variables, which yields $X_{12}(1,4,6)$. Note that this curve has the $Z_{2}$ singular point $X_{6}(2,3)$. The Euler number is obtained by combining the adjunction formula with the Riemann-Hurwitz formula: $\chi\left(C_{g}\right)=\left.\frac{(1+J)(1+4 J)(1+6 J) 12 J}{4 \cdot 6(1+12 J)}\right|_{J^{2}}-1 / 2+1 \cdot 1=0$. Hence there is $g=1$ hypermultiplet and the spectrum is conformal.

Recall that the above CY space $M$ with Euler number $\chi=-480$ exhibits several fibration structures. On the one hand it is an elliptic fibration over the Hirzebruch surface $\mathbf{F}_{2}$, which is itself a rational fibration with fiber $\mathbf{P}_{U}^{1}$ and base $\mathbf{P}_{S}^{1}$. At the same time the CY space is a $K 3$ fibration over the same base, hence the identification of the heterotic dilaton with $S$. We have as Kähler classes $S$, the size of the base $\mathbf{P}_{S}^{1}, U=\tilde{U}-\tilde{T}$ the size of the fiber $\mathbf{P}_{U}^{1}$ and $T=\tilde{T}$ the size of a curve in the elliptic fiber. Hence we have the nonvanishing intersections $C_{T T T}=8, C_{S T T}=2, C_{U T T}=4, C_{S T U}=1$, $C_{T U U}=2, \int c_{2} J_{T}=92, \int c_{2} J_{S}=24$ and $\int c_{2} J_{U}=36$. This fixes the integral basis. The mirror manifold $W$ has the following discriminant (we have rescaled $x=432 z_{t}, y=4 z_{s}$, $\left.y=4 z_{u}\right)$

$$
\Delta_{s} \Delta_{A} \Delta_{B}=(1-y)\left[(1-z)^{2}-y z^{2}\right]\left[\left((1-x)^{2}-z\right)^{2}-y z^{2}\right]
$$

which consists of three factors. $\Delta_{A}, \Delta_{B}$ are conifold loci. At $z=1, y=0, \Delta_{B} \neq 0$ there is a weakly coupled $S U(2)$ without matter [16]. We will be interested in the $S U(2)$ with an adjoint hypermultiplet, i.e. with a conformal spectrum, which arises at $y=1, \Delta_{A} \neq 0 \neq \Delta_{B}$, e.g. at $y=1, x=z=0$. To understand the rôle of the hypermultiplets it is interesting to contrast this model with the more generic realization of the $X_{24}(1,1,2,8,12) \mathrm{CY}$, which is given by an elliptic fibration over $\mathbf{F}_{0}$. As discussed in [36] both models are in the same moduli space. However in the $X_{24}(1,1,2,8,12)$ model the scalars in one hypermultiplet ${ }^{20}$ are set to a special value, which allows for

\footnotetext{
${ }^{19}$ Quantities with the tilde refer here to the heterotic spacetime moduli of $T^{2}$ in the compactification on $K 3 \times T^{2}$, i.e. $\tilde{U}$ is the complex structure modulus of $T^{2}$ and $\tilde{T}$ its Kähler modulus.

${ }^{20}$ This is the hypermultiplet related to the three-cycle which consists of the base $\mathbf{P}^{1}$ and a one-cycle on the elliptic fiber.
} 
the fibration structure of the divisor $F$. The linear reparameterisation at large coupling $T \rightarrow T, S \rightarrow S, U \rightarrow U-S$ relates the classical couplings. For the mirror of the $\mathbf{F}_{0}$ fibration the discriminant only factorizes into two factors:

$$
\Delta=\Delta_{A}^{\prime} \Delta_{B}^{\prime}=\left[(1-y)^{2}+(1-z)^{2}-1+y z\right]\left[(1-x)^{4}-2 x^{2}(1-x)^{2}(y+z)+x^{2}\left(y^{2}+z^{2}\right)\right]
$$

where $x, y, z$ are the coordinates on moduli space. Here we have a weakly coupled $S U(2)$ without matter at $z=1, y=0, \Delta_{B}^{\prime} \neq 0$ (e.g. for $x=0$ ) and at $y=1, z=0, \Delta_{B}^{\prime} \neq 0$ (e.g. for $x=0$ ) [35]. We can hence understand the deformation in the hypermultiplet moduli space away from the $X_{24}(1,1,2,8,12)$ Calabi-Yau as giving mass to the hypermultiplet in the adjoint at $y=1, x=z=0$.

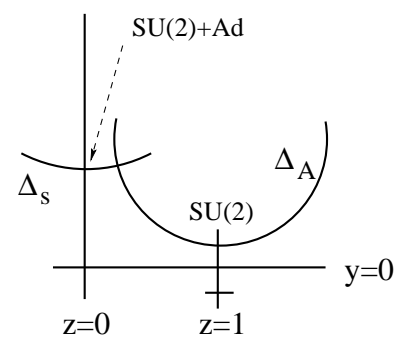

$\mathrm{F}_{2}$

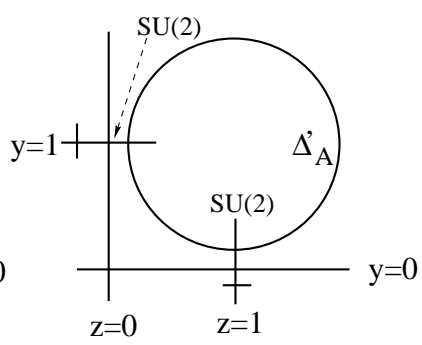

$\mathrm{F}_{0}$

Fig. 4 Slice of the quantum moduli space of the elliptic fibrations over $\mathbf{F}_{2}, \mathbf{F}_{0}$ at $x=0$ (local limit).

Let us now discuss the solution at $y=1, x=z=0$. As in equation (3.68) we have a unique solution $\Xi_{s}^{1}=1+\mathcal{O}(1)$. Furthermore the periods $\Xi_{s}^{2}, \Xi_{s}^{4}, \Xi_{s}^{5}, \Xi_{s}^{6}$ and $\Xi_{s}^{8}$ have the same $\log$ arithmic behaviour in $\log \left(z_{t}\right)$ and $\log \left(z_{u}\right)$ as (3.2). We can fix them uniquely by setting as many of the leading terms in the pure power series as possible to zerot Let us give the remaining solutions

$$
\begin{aligned}
& \left.\Xi_{s}^{3}=\frac{i}{\pi} \sqrt{w_{s}}\left(\frac{\arctan \left(\sqrt{-w_{s}}\right)}{i \sqrt{w_{s}}}+60 z_{t}+\mathcal{O}(2)\right)=a+\mathcal{O}\left(z_{t}, z_{u}\right)\right) \\
& \left.\Xi_{s}^{7}=\frac{X_{s}^{3}}{2 \pi i} \log \left(z_{t}\right)-\frac{\sqrt{w_{s}}}{2 \pi^{2}}\left(312 z_{t}+\mathcal{O}(2)\right)=a_{D}+\mathcal{O}\left(z_{t}, z_{u}\right)\right)
\end{aligned}
$$

The crucial difference to (3.68) is that the coordinate $w_{s}$ does not appear in the logarithm of the $a_{D}$ period. We can compute analytically the whole transformation matrix except

\footnotetext{
${ }^{21}$ We set the highest powers of $z_{t}, w_{s}, z_{u}$ in this order to zero.
} 
one numerical constant

$$
N_{s t r}=\left(\begin{array}{cccccccc}
1 & 0 & 0 & 0 & 0 & 0 & 0 & 0 \\
0 & 1 & 0 & 0 & 0 & 0 & 0 & 0 \\
0 & 0 & 1 & 0 & 0 & 0 & 0 & 0 \\
i A & 0 & -\frac{1}{2} & 1 & 0 & 0 & 0 & 0 \\
B_{-} & -4 i A & 0 & -2 i A & 1 & 0 & 0 & 0 \\
1 & -i A & 0 & 0 & 0 & \frac{1}{2} & 1 & 0 \\
2 & -2 i A & 0 & 0 & 0 & 1 & 0 & 0 \\
C & B_{+} & 0 & 2 & 0 & -i A & 0 & 1
\end{array}\right),
$$

where $A=\frac{\log (2)}{2 \pi}, B_{ \pm}=\frac{23}{6} \pm \frac{a}{(2 \pi i)^{2}}, a=.4804525$ and $C=\frac{63 i}{2} \frac{\zeta(3)}{\pi^{3}}+2 i A$. We see from this matrix that the flux generating

$$
W=n \Xi_{s}=n S
$$

is an integral Ramond-Flux w.r.t. the basis at large volume.

The scalar potential is extremely complicated. The behaviour near $z_{t}=z_{u}=w_{s}=0$ is determined by

$$
v \sim \frac{p_{8}^{0}+p_{8}^{1} \sqrt{\left|w_{s}\right|}+p_{9}^{1}}{q_{9}+q_{10}}
$$

where $p_{d}^{i}$ and $q_{d}$ are generic homogeneous polynomials in $\left(\log \left(\left|z_{t}\right|\right), \log \left(\left|z_{u}\right|\right)\right)$ of the indicated degree $d$. In particular the leading logarithmic term

$$
v \sim-2 \pi \frac{4 \log \left(\left|z_{u}\right|\right)+9 \log \left(\left|z_{t}\right|\right)}{\log \left(\left|z_{u}\right|\right)^{2}+6 \log \left(\left|z_{t}\right|\right) \log \left(\left|z_{3}\right|\right)+9 \log \left(\left|z_{t}\right|\right)^{2}}
$$

is independent of $w_{s}$. The potential has a minimum at $z_{t}=0$ as well as at $z_{u}=0$ with vanishing energy. For fixed values of $z_{t}, z_{u}$ the potential can be analyzed to 10-th order in $\sqrt{w_{s}}$ and we found a series $v \sim \sum_{i=0} a_{i}\left(z_{t}, z_{u}\right)\left(\left|w_{s}\right|\right)^{\frac{i}{2}}$ whose coefficients $a_{i}$ go to zero if $z_{t}$ or $z_{u}$ goes to zero.

Similarly to the situation above (3.74), where two periods vanish with a square root, it was found in [31] at the point $w=0$, where tensionless strings arise, that two dual

periods occur which start with $w^{\frac{1}{6}}$ and $w^{\frac{5}{6}}$. Therefore we expect for fluxes aligned with a vanishing direction a similar behavior of the scalar potential in the direction of $w$, i.e. a power series in $w^{\frac{n}{6}}$ with $n \in \mathbf{Z}$.

\section{Type II Vacua with Non-vanishing Ramond and NS Fluxes}

In sect. 3.7 on the discussion of the Seiberg-Witten limit NS-fluxes have already emerged. We now want to study in little more detail the possible effects of non-vanishing NS-fluxes 
for the vacuum structure of type IIB strings. Since the superpotential is covariant under the type IIB $S L(2, \mathbf{Z})$ duality symmetry (see eq.(2.33)), the scalar potential is invariant under this symmetry. Hence we expect to find non-trivial minima, where the field $\tau$ is stabilized. The following discussion will be performed in the STU model, where we can solve the minimization conditions explicitly. As we will see now, some of our previous conclusions concerning the vector multiplets are modified by the presence of NS fluxes. In particular it seems possible that the contribution of the $\mathrm{R}$ fluxes is precisely balanced by the NS fluxes, such that supersymmetric minima of the scalar potential are possible at non-degenerate points in the CY moduli space, i.e. at finite value for the $S$-field. It is worth pointing out that in the example we are going to discuss the NS and Ramond flux vectors are non-local w.r.t. each other. Nevertheless we will find that the ground state of the model is fully $\mathcal{N}=2$ supersymmetric.

For simplicity we consider the perturbative heterotic prepotential with only three moduli $S, T$ and $U$ and $S \rightarrow \infty: F=i\left(X^{0}\right)^{2} S T U$. After the symplectic transformation

$$
S \rightarrow F_{S}, \quad F_{S} \rightarrow-S
$$

the period vector has the form

$$
\left(\tilde{X}^{I}, \tilde{F}_{I}\right)=(1,-T U, i T, i U,-i S T U, i S, S U, S T)
$$

One recognizes that the periods $\tilde{X}^{I}$ are algebraically dependent. Now we choose the flux vectors such that all electric NS fluxes and also all magnetic Ramond fluxes are zero, i.e. $e_{I}^{1}=m^{2 I}=0$. In addition, in order to be able to balance the $\mathrm{R}$ fluxes against the NS fluxes we choose them to be parallel, i.e. we impose the following condition:

$$
e_{I}^{2} / p=\left(l_{2},-n_{2}, n_{1},-l_{1}\right), \quad m^{1 I} / q=\left(-n_{2}, l_{2},-l_{1}, n_{1}\right) .
$$

In fact, these two flux vectors are mutually non-local, $m \times e=m^{1 I} e_{I}^{2}-m^{2 I} e_{I}^{1}=$ $-2 p q\left(l_{1} n_{1}+l_{2} n_{2}\right) \neq 0$. With this choice the superpotential becomes

$$
W=(p+i q S \tau)\left(l_{2}-i l_{1} U+i n_{1} T-n_{2} U T\right),
$$

It is straightforward to see that the scalar potential has a $\mathcal{N}=2$ supersymmetry preserving minimum with zero cosmological constant; specifically the supersymmetry conditions,

$$
W_{S}=W_{T}=W_{U}=W_{\tau}=0, \quad \text { and } \quad W=0,
$$

have the following solution:

$$
T_{\min }=\sqrt{\frac{l_{1} l_{2}}{n_{1} n_{2}}}, \quad U_{\min }=\sqrt{\frac{l_{2} n_{1}}{l_{1} n_{2}}}, \quad S_{\min } \tau_{\min }=i \frac{p}{q} .
$$

So we see that supersymmetry preserving solutions are possible for finite heterotic dilaton field $S$, as well as for finite type IIB dilaton $\tau$. Insisting on large $S$ and on large $\tau$ implies that one has to ensure that $p \gg q$. 


\section{Summary}

In this paper we have provided a detailed investigation of the vacuum structure of type IIA/B compactifications on Calabi-Yau spaces in the presence of H-fluxes. These H-fluxes lift the vacuum degeneracy in the Calabi-Yau moduli space. For aligned fluxes, local minima of the scalar potential with space-time supersymmetry and vanishing cosmological constant are found at several degeneration points of the Calabi-Yau space. However partial supersymmetry at these minima seems to be impossible in local supergravity due to the dilaton dependence of the effective superpotential, such that the degeneration points always exhibit full $\mathcal{N}=2$ supersymmetry. Away from the degeneration points supersymmetry is generically broken, and we expect in general local minima of the scalar potential with broken supersymmetry. We also examined the vacuum structure of some Calabi-Yau spaces (quintic, sextic) at certain rational, but non-singular points of enlarged symmetry (Gepner points), and found that there are no supersymmetric solutions at this points. However at the moment we cannot completely exclude supersymmetric vacua at non-singular, rational points for other Calabi-Yau spaces.

This discussion can be extended in several ways. For example it would be interesting to map the type IIA/B H-fluxes to dual heterotic or type I string compactifications. There the H-fluxes will correspond to background electric or magnetic fields in the internal directions [32] (for non-supersymmetric string compactifications with background Ffields see [33]). Similarly it would be nice to see [32] how the type IIA/B H-fluxes at the Calabi-Yau singularities can be mapped to dual brane configurations using the duality [34 between the Calabi-Yau geometrical engineering and the Hanany-Witten approach.

\section{Acknowledgements}

We would like to thank Jan Louis for many valuable discussions and for collaboration on the initial stage of this project. Discussions with K. Behrndt, G. Dell' Agata and G. Lopes-Cardoso are also acknowledged. This work is supported by the European Commission RTN programme HPRN-CT-2000-00131 and by GIF - the German-Israeli Foundation for Scientific Research. D.L. and S.T. thank the Erwin-Schroedinger-Institute in Vienna, where this project was started, for hospitality.

\footnotetext{
${ }^{22}$ This was already conjectured for the flux related to $X^{0}$ in [1].
} 


\section{Appendix: The perturbative heterotic limit with general flux vector}

In this appendix we like to solve in some detail the conditions of unbroken supersymmetry within the perturbative heterotic limit for general flux vector $\left(e_{I}, m^{I}\right)$. The corresponding prepotential is given in eq.(3.20). After the symplectic transformation (4.1) the period vector has the form

$$
\left(\tilde{X}^{I} ; \tilde{F}_{I}\right)=\left(1,-T^{a} \eta_{a b} T^{b}, i T^{a} ;-i S T^{a} \eta_{a b} T^{b}, i S, 2 S \eta_{a b} T^{b}\right)
$$

One recognizes that the periods $\tilde{X}^{I}$ are algebraically dependent. The symplectic transformation (4.1) exchanges the IIA 2-cycle $\mathcal{C}_{1}^{(2)} \sim \operatorname{vol}\left(P_{b}^{1}\right)$ by its dual 4-cycle $\mathcal{C}_{1}^{(4)}$, namely the whole $K 3$-fibre. This amounts in exchanging $H_{R}^{(4)}$ by $H_{R}^{(2)}$ in the $S$-field direction and vice versa, and we denote the corresponding fluxes as

$$
\tilde{e}_{1}=m^{1}, \quad \tilde{m}^{1}=e_{1}
$$

Seen from the heterotic point of view, the symplectic basis eq.(5.1) is the most natural one since $\tilde{X}^{I}$ are the perturbative periods associated to the electric $U(1)$, whereas the $\tilde{F}_{I}$ are the non-perturbative magnetic $U(1)$ periods.

For simplicity consider the case of only three moduli fields $S, T$ and $U$. The classical period vector is then

$$
\left(\tilde{X}^{I}, \tilde{F}_{I}\right)=(1,-T U, i T, i U,-i S T U, i S, S U, S T)
$$

The holomorphic superpotential is then given as

$$
W=e_{0}+\tilde{e}_{1} T U+i e_{2} T+i e_{3} U+i m^{0} S T U+i \tilde{m}^{1} S-m^{2} S U-m^{3} S T
$$

and the Kähler potential take the well known form

$$
K=-\log [(S+\bar{S})(T+\bar{T})(U+\bar{U})]
$$

In order to keep the notation as simple as possible we will omit from now on in this chapter the tilde on $e_{1}$ and $m^{1}$.

The perturbative duality transformations $\left.S L(2, \mathbf{Z})_{T} \otimes S L(2, \mathbf{Z})_{U}\right) \times \mathbf{Z}_{2}^{T \leftrightarrow U}$ act as symplectic transformations on the period vector as

$$
\left(\begin{array}{c}
\tilde{X}^{I} \\
\tilde{F}_{I}
\end{array}\right) \rightarrow \Gamma\left(\begin{array}{c}
\tilde{X}^{I} \\
\tilde{F}_{I}
\end{array}\right)=\left(\begin{array}{cc}
U & 0 \\
0 & U^{T,-1}
\end{array}\right)\left(\begin{array}{c}
\tilde{X}^{I} \\
\tilde{F}_{I}
\end{array}\right)
$$


The generalized Kähler function $e^{G}=e^{K}|W|^{2}$ is invariant under symplectic transformations (5.6) provided the quantum numbers are redefined by $\left(m^{I},-e_{I}\right) \rightarrow\left(m^{I},-e_{I}\right) \Gamma^{T}$. Note that under the modular transformations $S L(2, \mathbf{Z})_{T} \otimes S L(2, \mathbf{Z})_{U}$ the superpotential transforms as a modular function of modular weight -1 , as required for the modular invariance of $G$ [37]:

$$
T \rightarrow \frac{a T-i b}{i c T+d}: \quad W \rightarrow \frac{W}{i c T+d}
$$

Now let us look for points of which preserve $\mathcal{N}=1$ supersymmetry, i.e. we look for solutions of the equation

$$
D_{a} W=0 \rightarrow e^{K / 2}\left(K_{a} W+W_{a}\right)=0, a=S, T, U
$$

This problem was already solved [20] using the attractor equations (2.52), and the full solution of $(5.8)$ is

$$
\begin{aligned}
& S_{\text {Susy }}=i \frac{e \cdot m}{\langle m, m\rangle}+\sqrt{\frac{\langle e, e\rangle}{\langle m, m\rangle}-\frac{(e \cdot m)^{2}}{\langle m, m\rangle^{2}}}, \\
& T_{\text {Susy }}=i \frac{e^{\prime} \cdot m^{\prime}}{\left\langle m^{\prime}, m^{\prime}\right\rangle}+\sqrt{\frac{\left\langle e^{\prime}, e^{\prime}\right\rangle}{\left\langle m^{\prime}, m^{\prime}\right\rangle}-\frac{\left(e^{\prime} \cdot m^{\prime}\right)^{2}}{\left\langle m^{\prime}, m^{\prime}\right\rangle^{2}}}, \\
& U_{\text {Susy }}=i \frac{e^{\prime \prime} \cdot m^{\prime \prime}}{\left\langle m^{\prime \prime}, m^{\prime \prime}\right\rangle}+\sqrt{\frac{\left\langle e^{\prime \prime}, e^{\prime \prime}\right\rangle}{\left\langle m^{\prime \prime}, m^{\prime \prime}\right\rangle}-\frac{\left(e^{\prime \prime} \cdot m^{\prime \prime}\right)^{2}}{\left\langle m^{\prime \prime}, m^{\prime \prime}\right\rangle^{2}}},
\end{aligned}
$$

where

$$
\begin{aligned}
\langle e, e\rangle & =2 e_{0} e_{1}+2 e_{2} e_{3}, \\
\langle m, m\rangle & =2 m^{0} m^{1}+2 m^{2} m^{3}, \\
e \cdot m & =e_{0} m^{0}+e_{1} m^{1}+e_{2} m^{2}+e_{3} m^{3} .
\end{aligned}
$$

The exchange symmetries $S \leftrightarrow T$ and $S \leftrightarrow U$ map the vectors $e, m$ to vectors $e^{\prime}, m^{\prime}$ and $e^{\prime \prime}, m^{\prime \prime}$.

The function $e^{G}$ at the supersymmetric point (5.9) takes then the following value

$$
\left.m_{3 / 2}^{2}\right|_{\text {Susy }}=\left.e^{G}\right|_{\text {Susy }}=\sqrt{\langle e, e\rangle\langle m, m\rangle-(e \cdot m)^{2}}=\left.\langle m, m\rangle \operatorname{Re} S\right|_{\text {Susy }} .
$$

From (5.11) one can easily read off that supersymmetric minima of the scalar potential $v$ with $v=0$ at the minimum, i.e. $\left.W\right|_{\text {Susy }}=\left.e^{G}\right|_{\text {Susy }}=0$, are possible for certain choices of fluxes. The first possibility for solving these two conditions is given by choosing parallel electric and magnetic fluxes:

$$
e_{I} / p=\left(l_{2},-n_{2}, n_{1},-l_{1}\right), \quad m^{I} / q=\left(-n_{2}, l_{2},-l_{1}, n_{1}\right) .
$$


This implies that

$$
\begin{aligned}
\langle e, e\rangle & =-2 p^{2} n^{T} l, \\
\langle m, m\rangle & =-2 q^{2} n^{T} l, \\
e \cdot m & =-2 p q n^{T} l .
\end{aligned}
$$

With this choice the superpotential is

$$
W=(p+i q S)\left(l_{2}-i l_{1} U+i n_{1} T-n_{2} U T\right)
$$

and therefore $\left.e^{G}\right|_{\text {Susy }}=0$. However for this class of solutions $\operatorname{Re} S$ is zero, so one is driven to strong coupling which is not consistent with assumption of having a large $S$-field.

The second class of supersymmetric solutions is given by only four non-vanishing flux quantum numbers, namely the cases of purely electric $\left(m^{I}=0\right)$ and purely magnetic $\left(e_{I}=0\right)$ charges. In both cases the moduli $T_{\text {Susy }}$ and $U_{\text {Susy }}$ are generically finite. For consistency we have to require in addition that $S_{\min }=\infty$. This constraint is satisfied for the purely electric solutions with $m^{I}=0$. This is precisely the case with aligned fluxes and superpotential eq.(3.21). On the other hand the case with $e_{I}=0$ would imply strong coupling with $S_{\text {Susy }}=0$.

Next let us consider the effects of one-loop corrections $h\left(T^{a}\right)$ to the heterotic prepotential. Due to the required embedding of the perturbative $T$-duality group into the $\mathcal{N}=2$ symplectic transformations, it follows [38, 39] that the heterotic one-loop prepotential $h\left(T^{a}\right)$ must obey well-defined transformation rules under this group. This becomes clear if one considers the action of the one-loop T-duality transformations on the period vector (5.1):

$$
\left(\begin{array}{c}
\tilde{X}^{I} \\
i \tilde{F}_{I}
\end{array}\right) \rightarrow \Gamma_{1-\text { loop }}\left(\begin{array}{c}
\tilde{X}^{I} \\
i \tilde{F}_{I}
\end{array}\right)=\left(\begin{array}{cc}
U & 0 \\
V & U^{T,-1}
\end{array}\right)\left(\begin{array}{c}
\tilde{X}^{I} \\
i \tilde{F}_{I}
\end{array}\right)
$$

where the matrix $V$ encodes the quantum corrections. Therefore the one-loop transformation rule of symplectic quantum numbers implied by the general formula $(m,-e) \rightarrow$ $(m,-e) \Gamma^{T}$ is

$$
e \rightarrow U^{T,-1} e-V m, \quad m \rightarrow U m
$$

It follows that the superpotential $W$ is still transforms with modular weight -1 under $S L(2, \mathbf{Z})_{T}$ and $S L(2, \mathbf{Z})_{U}$.

In the presence of the heterotic one loop correction $h\left(T^{a}\right)$ the period vector eq.(5.1) will 
be modified as follows:

$$
\left.\left(\tilde{X}^{I} ; \tilde{F}_{I}\right)\right|_{1-\mathrm{loop}}=\left(1, T^{a} \eta_{a b} T^{b}, i T^{a} ; i S, i S T^{a} \eta_{a b} T^{b}+2 i h\left(T^{a}\right)-i T^{a} \frac{\partial h}{\partial T^{a}},-S T^{a}+\frac{\partial h}{\partial T^{a}}\right)
$$

One recognizes, that the periods $\tilde{X}^{I}$ do not receive any quantum corrections and are still algebraically dependent. Hence the superpotential with aligned fluxes is the same as in the classical case eq.(3.21), and we therefore expect the same vacuum structure as in the classical limit for aligned fluxes. The one-loop quantum corrections only result in a simple modification of the Kähler potential which can be absorbed by the invariant dilaton field $S_{\text {invar }}$ 38]. This field $S_{\text {invar }}$ is the true coupling constant at one loop, and $e^{K_{1-\text { loop }} / 2}$ is proportional to $S_{\text {invar }}^{-1}$.

Let us consider in a little bit more detail the complete superpotential

$$
W=e_{I} \tilde{X}^{I}+\left.m^{I} \tilde{F}_{I}\right|_{1-\mathrm{loop}}
$$

which includes the one-loop term $h\left(T^{a}\right)$ in $\left.\tilde{F}_{I}\right|_{1-\text { loop. }}$. The supersymmetry equations $D_{a} W=0, W=0$ will still allow for non-trivial solutions which however cannot be expressed any more in closed form like in eq.(5.9) for non-vanishing $m^{I}$. Nevertheseless one can derive an all order expression for $m_{3 / 2}$ at the supersymmetric, stationary points, as it was proven in the context of $\mathcal{N}=2$ black hole solutions. This expression has simply the form

$$
\left.m_{3 / 2}^{2}\right|_{\text {Susy }}=\left.e^{G}\right|_{\text {Susy }}=\pi \operatorname{Re} S_{\text {invar }}\langle m, m\rangle,
$$

where for the case of $N_{V}-1$ fields $T^{a},\langle m, m\rangle$ is defined as

$$
\langle m, m\rangle=m^{0} m^{1}+m^{a} \eta_{a b} m^{b} .
$$

$\left(\langle m, m\rangle\right.$ is an invariant of the T-duality group $S O\left(2, N_{V}-1, \mathbf{Z}\right)$.) Thus the influence of all perturbative one-loop effects to the superpotential is contained in $\operatorname{Re} S_{\text {invar }}$. Hence, demanding $\left.e^{G}\right|_{\min }=0$ at $\operatorname{Re} S_{\text {invar }}=\infty$, one has to set again $\langle m, m\rangle=0$.

\section{References}

[1] J. Polchinski and A. Strominger, Phys. Lett. B388 (1996) 736, hep-th/9510227.

[2] J. Michelson, Nucl. Phys. B495 (1997) 127, hep-th/9610151.

[3] T.R. Taylor and C. Vafa, Phys. Lett. B474 (2000) 130, hep-th/9912152.

[4] P. Mayr, Nucl. Phys. B593 (2000) 99, hep-th/0003198. 
[5] A. Strominger, Nucl. Phys. B274 (1986) 274.

[6] S. Ferrara, C. Kounnas, D. Lüst and F. Zwirner, Nucl. Phys. B365 (1991) 431.

[7] I. Antoniadis, H. Partouche and T.R. Taylor, Phys. Lett. B372 (1996) 83, hepth/9512006.

[8] S. Ferrara, L. Girardello and M. Porrati, Phys. Lett. B366 (1996) 155, hepth/9510074;

S. Ferrara, L. Girardello and M. Porrati, Phys. Lett. B376 (1996) 275, hepth/9512180.

[9] S. Ferrara and R. Kallosh, Phys. Rev. D54 (1996) 1514, hep-th/9602136;

S. Ferrara and R. Kallosh, Phys. Rev. D54 (1996) 1525, hep-th/9603090.

[10] G. Moore, Attractors and arithmetic, hep-th/9807056;

G. Moore, Arithmetic and attractors, hep-th/9807087.

[11] S. Gukov, C. Vafa and E. Witten, Nucl. Phys. B584 (2000) 69, hep-th/9906070;

S. Gukov, Nucl. Phys. B574 (2000) 169, hep-th/9911011.

[12] M. Haack, J. Louis and M. Marquart, Type IIA and heterotic string vacua in D $=2$, hep-th/0011075.

[13] B. de Wit and A. Van Proeyen, Nucl. Phys. B 245 (1984) 89.

[14] S. Ferrara and A. Strominger, in Strings '89, eds. R. Arnowitt, R. Bryan, M.J. Duff, D.V. Nanopoulos and C.N. Pope (World Scientific, 1989), p. 245;

A. Strominger, Commun. Math. Phys. 133 (1990) 163;

L.J. Dixon, V.S. Kaplunovsky and J. Louis, Nucl. Phys. B329 (1990) 27;

P. Candelas and X.C. de la Ossa, Nucl. Phys. B355 (1991) 455;

L. Castellani, R. D' Auria and S. Ferrara, Phys. Lett. B 241 (1990) 57; Cl.Q. Grav. 7 (1990) 1767;

R. D'Auria, S. Ferrara and P. Fré, Nucl. Phys. B359 (1991) 705;

B. de Wit and A. Van Proeyen, in Quaternionic Structures in Mathematics and Physics, ILAS/FM-6/1996 (Sissa, Trieste) hep-th/9505097; Nucl. Phys. B (Proc. Suppl.) 45B,C (1996) 196.

[15] B. de Wit, P. G. Lauwers, R. Philippe, S.-Q. Su and A. Van Proeyen, Phys. Lett. 134B (1984) 37. 
[16] S. Kachru, A. Klemm, W. Lerche, P. Mayr and C. Vafa, Nucl. Phys. B459 (1996) 537, hep-th/9506112.

[17] A. Klemm and S. Theisen, Nucl. Phys. B389 (1993) 153, hep-th/9205041; A. Font, Nucl. Phys. B391 (1993) 358, hep-th/9203084.

[18] L. Andrianopoli, M. Bertolini, A. Ceresole, R. D'Auria, S. Ferrara and P. Fre, Nucl. Phys. B476 (1996) 397, hep-th/9603004.

[19] A. Ceresole, R. D'Auria, S. Ferrara and A. Van Proeyen, Nucl. Phys. B444 (1995) 92, hep-th/9502072.

[20] G. L. Cardoso, D. Lüst and T. Mohaupt, Phys. Lett. B388 (1996) 266, hepth/9608099.

[21] K. Behrndt, G. Lopes Cardoso, B. de Wit, R. Kallosh, D. Lüst and T. Mohaupt, Nucl. Phys. B488 (1997) 236, hep-th/9610105.

[22] D. Lüst, String vacua with $N=2$ supersymmetry in four dimensions, hepth/9803072.

[23] P. Candelas, X. C. de la Ossa, P. S. Green and L. Parkes, Nucl. Phys. B359 (1991) 21.

[24] S. Hosono, A. Klemm, S. Theisen and S.-T. Yau, Commun. Math. Phys. 167 (1995) 301, hep-tn/9308122 and Nucl. Phys. B433, 501 (1995), hep-th/9406055.

[25] P. Candelas, X. C. de la Ossa, A. Font, S. Katz and D. Morrison, Nucl. Phys. B416 (1994) 481, hep-th/9308083.

[26] A. Strominger, S.-T. Yau and E. Zaslow, Nucl. Phys. B479 (1996) 243, hepth/9606040.

[27] N. Seiberg and E. Witten, Nucl. Phys. B426 (1994) 19, hep-th/9407087.

[28] A. Klemm and P. Mayr, Nucl. Phys. B469 (1996) 37, hep-th/9601014;

S. Katz, D. Morrison and M.R. Plesser, Nucl. Phys. B477 (1996) 105, hepth/9601108.

[29] A. Strominger, Nucl. Phys. B451 (1995) 96, hep-th/9504090.

[30] K. Behrndt, D. Lüst and W. Sabra, Phys. Lett. B418 (1998) 303, hep-th/9708065. 
[31] A. Klemm, P. Mayr and C. Vafa, BPS states of exceptional non-critical strings, hep-th/9607139.

[32] Work in progress.

[33] C. Bachas, A way to break supersymmetry, hep-th/9503030;

R. Blumenhagen, L. Görlich, B. Körs and D. Lüst, JHEP 0010 (2000) 006, hepth/0007024;

R. Blumenhagen, L. Görlich, B. Körs and D. Lüst, Magnetic flux in toroidal type I compactifications, hep-th/0010198;

C. Angelantonj, I. Antoniadis, E. Dudas and A. Sagnotti, Phys. Lett. B489 (2000) 223, hep-th/0007090;

C. Angelantonj and A. Sagnotti, Type-I vacua and brane transmutation, hepth/0010279;

G. Aldazabal, S. Franco, L.E. Ibanez, R. Rabadan and A.M. Uranga, Chiral string compactifications from intersecting branes, hep-th/0011073.

[34] A. Karch, D. Lüst and D. Smith, Nucl. Phys. B533 (1998) 348, hep-th/9803232.

[35] S. Katz, A. Klemm and C. Vafa, Nucl. Phys. B497 (1997) 173, hep-th/9609239.

[36] D. R. Morrison and Cumrun Vafa, Nucl.Phys. B476 (1996) 437-469, hep-th/9603161.

[37] S. Ferrara, D. Lüst, A. Shapere and S. Theisen, Phys. Lett. B225 (1989) 363;

A. Font, L.E. Ibanez, D. Lüst and F. Quevedo, Phys. Lett. B245 (1990) 401.

[38] B. de Wit, V. Kaplunovsky, J. Louis and D. Lüst, Nucl. Phys. B451 (1995) 53, hep-th/9504006.

[39] I. Antoniadis, S. Ferrara, E. Gava, K. S. Narain and T. R. Taylor, Nucl. Phys. B447 (1995) 35, hep-th/9504034.

[40] D.-E. Diaconescu and C. Römelsberger, Nucl.Phys. B574 (2000) 245, hepth/9910172.

[41] S. Hosono, Local Mirror Symmetry and Type IIA Monodromy of Calabi-Yau manifolds, Adv. Theor. Math. Phys. 4 (2000), hep-th/0007071.

[42] P. Mayr, Phases of Supersymmetric D-branes on Kähler Manifolds and the McKay correspondence, hep-th/0010223.

[43] P. Candelas, A. Font, S. Katz and D. R. Morrison, Nucl. Phys. B429 (1994) 626, hep-th/9403187. 